\title{
On the ichnofossil Treptichnus pedum: inferences from the Nagaur Sandstone, Marwar Supergroup, India
}

\author{
Mukund Sharma, Shamim Ahmad, Santosh K. Pandey \& Kuldeet Kumar
}

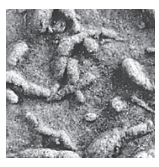

Sandstone-mudstone interfaces offer an excellent medium for the preservation of the burrows. This study discusses the morphology, ecological niche and correlation based of 291 Treptichnus pedum specimens of the lower Cambrian Nagaur Sandstone, western Rajasthan, India. Petrological attributes of the host sediment are also addressed. Statistical analyses of size (length, width and gap of each segment of $T$. pedum) were conducted to determine the inter-specific variation. Data suggest that the entire Nagaur assemblage was made by one major group of makers. Overall morphology suggests that these burrows were probably made for shelter and trapping small epibenthic or endobenthic organisms. An overview of the global occurrence of $T$. pedum shows broad environment tolerance. The Nagaur specimens are compared with various recorded occurrences of $T$. pedum to assess the nature of the plausible producer organism, its behaviour and taphonomical aspects. - Key words: Treptichnus, Priapulid, Cambrian, Nagaur Group, Marwar Supergroup.

Sharma, M., Ahmad, S., Pandey, S.K. \& Kumar, K. 2018. On the ichnofossil Treptichnus pedum: inferences from the Nagaur Sandstone, Marwar Supergroup, India. Bulletin of Geosciences 93(3), 305-325 (10 figures, 3 tables). Czech Geological Survey, Prague. ISSN 1214-1119. Manuscript received October 7, 2017; accepted in revised form April 24, 2018; published online July 13, 2018; issued August 20, 2018.

Mukund Sharma, Centre of Advanced Study, Department of Geology, Banaras Hindu University, Varanasi-221005, India \& Birbal Sahni Institute of Palaeosciences, 53 University Road,Lucknow-226007, India; mukund_sharma@, bsip.res.in - Shamim Ahmad \& Santosh K. Pandey, Birbal Sahni Institute of Palaeosciences, 53 University Road, Lucknow-226007, India; shamimfragerence@gmail.com • Kuldeep Kumar, Department of Economics and Statistics, Bond University, Gold Coast, Queensland 4229, Australia

Trace fossils provide key insights into benthic faunas and their distribution in space and time (Seilacher 2007). They not only document the lifestyle of the producers but also help in the interpretation of the associated environments. In the absence of geochronological data, certain trace/ body fossils are critical for determining the age of fossilbearing litho units, as well as depositional environment and palaeoecology. The Precambrian-Cambrian boundary and its Global Standard Stratotype Section and Point (GSSP) have been established at the base of the trace fossil Treptichnus pedum zone at Fortune Head, Newfoundland (Narbonne et al. 1987, Brasier et al. 1994, Landing 1994). Previously known as Phycodes pedum (Seilacher, 1955), the First Appearance Datum (FAD) of Treptichnus pedum is accepted as the index fossil representing the Precambrian-Cambrian boundary and has been reported from several Precambrian-Cambrian transitional successions worldwide (Seilacher 2007, Buatois 2018). Formed possibly by priapulid worms (Vannier et al. 2010), T. pedum is essentially a branching, feeding burrow structure resulting from systematic probing and backfilling. Priapulids are basically considered as endobenthic predators (Vannier et al. 2010), suggesting that T. pedum may indicate the presence of predation behaviour at the
Precambrian-Cambrian boundary. Morphologically these worm burrows are subdivided into modular segments, resembling buds on a twig, which follow a straight, sinusoidal or coiled course. They arguably represent the first record of complex metazoan behaviour at the onset of the Cambrian (Grotzinger et al. 1995, Jensen et al. 2006, Mángano et al. 2012). The burrowing method gradually became more complex, i.e. from horizontal to vertical in nature during this period as the priapulids probed deeper into the sediment in various ways. Such patterns distinguish it from other ordinary burrows, i.e. subhorizontal burrows produced in the sub-surface (Vannier et al. 2010).

The Nagaur Group, as exposed at the Dulmera section (Fig. 1A, B), is well known for its trace fossil content (Kumar \& Pandey 2008, 2010; Sharma \& Pandey 2011; Srivastava 2012a, b; Singh et al. 2013, 2014a; Ahmad \& Kumar, 2014; Pandey et al. 2014). Except a solitary claim by Singh et al. (2013), no convincing body fossil has yet been documented to date. Due to limited exposure of the Nagaur Sandstone, the FAD for the earlier reported T. pedum could not be assigned to any specific stratum at the Dulmera section (Srivastava 2012a). Futhermore, as previously pointed out with respect to Indian sections 
(Hughes et al. 2013, Hughes 2016), T. pedum has a stratigraphical range that extends from the base of the Cambrian into the Early Ordovician and so, its presence is not diagnostic of any particular age within the Cambrian. In this paper, we provide the details on the morphology, ecological niche, and distribution; petrological attributes of the host sediment are also provided. Treptichnus pedum reported from the Dulmera section is also evaluated for the possibility of the unit representing the FAD in the Nagaur Formation. The behaviour of the organism producing these burrows, as well as taphonomy of the ichnofossils, is also discussed.

\section{General Geology and Age}

The $1000 \mathrm{~m}$ thick Marwar Supergroup is represented by argillo-arenaceous and carbonates facies in the Jodhpur-Khatu-Bikaner-Phalodi areas of the western Rajasthan (Pareek 1984). The lithologies represented are conglomerate, sandstone, siltstone, shale, dolomite and limestone. The Marwar Supergroup has been stratigraphically divided into the three groups (Table. 1). In stratigraphically ascending order, these are the Jodhpur Group, the Bilara Group and the Nagaur Group. These groups are further subdivided into different formations. The Jodhpur and Nagaur groups are arenaceous to argillaceous, whereas the Bilara Group is mainly calcareous in nature. The Marwar succession rests unconformably above the Malani Igneous Suite that is dated $771 \pm 5 \mathrm{Ma}$ (Gregory et al. 2009). The Tunklian Sandstone is the youngest formation of the Marwar Supergroup. The underling Nagaur Sandstone has yielded the ichnofossils discussed in the present paper. Laser Ablation-Inductively Coupled Plasma-Mass Spectrometry (LA-ICP-MS) analysis calibrations of the detrital zircons recovered from the Nagaur Sandstone yielded 540 Ma ages (McKenzie et al. 2011), which is the maximum age of the Nagaur Sandstone. Pandey et al. (2014) and Singh et al. (2014a) considered that the ichnofossil assemblage recorded from the Nagaur Sandstone belongs to Stage 2 of the Terreneuvian Epoch. However, the beds containing these trace fossils are not conformably overlain by diagnostic short-ranged earliest Cambrian fossils and there are no convincing evidence that these sediments are Stage 2. Hughes (2016) considered these specimens to indicate Cambrian Series 2, late Stage 4 age ( $\sim 509$ Ma age) for the Dulmera outcrops. Therefore, the minimum age of the Nagaur Sandstone is constrained to be $\sim 509$ Ma.

\section{Phycodes pedum to Treptichnus pedum: A précis}

Phycodes Richter, 1850 and Treptichnus Miller, 1889 are two ichnogenera established more than a century ago. Seilacher (1955) erected the ichnospecies Phycodes pedum (pedum = bishop's crosier) from the Khussak Formation of the Salt Range which is early in the Cambrian Stage 4 ( $\sim 514 \mathrm{Ma})$ (Hughes 2016). This ichnotaxon-shows the advent of complex behaviour and anatomy at the beginning of the Cambrian. Seilacher reconstructed Phycodes pedum as a flat ' $U$ ' tube of which one end was fixed and the other end was gradually increased by probe on the curved path emerging on the surface. In the original discussion, he did not state whether the expanding ' $U$ ' shaped tube was inferred or observed. An assessment of illustrations provided with the description (Seilacher 1955, p. 387, fig. $4 \mathrm{~g}$ ) suggests that $P$. pedum was formed by the addition of segments in a treptichian manner. A weakly developed alternation of direction is seen also in the holotype of Phycodes pedum and therefore, the ichnogenus was later placed in Treptichnus (see Jensen 1997, Jensen \& Grant 1998, Jensen et al. 1998 for discussions).

Table 1. Generalized stratigraphic succession of the Marwar Supergroup (after Pareek 1984 and Chauhan et al. 2004).

\begin{tabular}{|c|c|c|c|}
\hline Age & Supergroup & Group & Formation \\
\hline \multicolumn{3}{|l|}{ Permo-Carboniferous } & Bap Boulder Bed \\
\hline \multicolumn{4}{|c|}{ Unconformity } \\
\hline \multirow{3}{*}{ 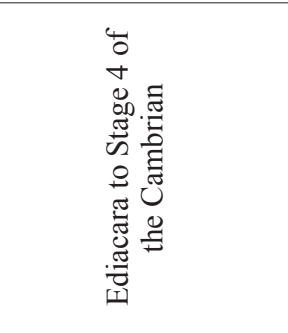 } & \multirow{3}{*}{ 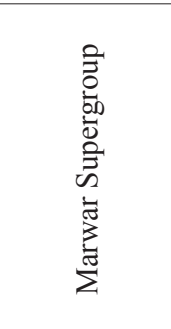 } & $\begin{array}{l}\text { Nagaur Group } \\
(75-500 \mathrm{~m})\end{array}$ & $\begin{array}{l}\text { Tunklian Sandstone } \\
\text { Nagaur Sandstone }\end{array}$ \\
\hline & & $\begin{array}{l}\text { Bilara Group } \\
(100-300 \mathrm{~m})\end{array}$ & $\begin{array}{l}\text { Pondlo Dolomite } \\
\text { Gotan Limestone } \\
\text { Dhanapa Dolomite }\end{array}$ \\
\hline & & $\begin{array}{l}\text { Jodhpur Group } \\
(125-240 \mathrm{~m})\end{array}$ & $\begin{array}{l}\text { Girbhakar Sandstone } \\
\text { Sonia Sandstone } \\
\text { Pokaran Boulder Bed }\end{array}$ \\
\hline \multicolumn{4}{|c|}{ Unconformity } \\
\hline 779-681 Ma & & Malan & \\
\hline
\end{tabular}




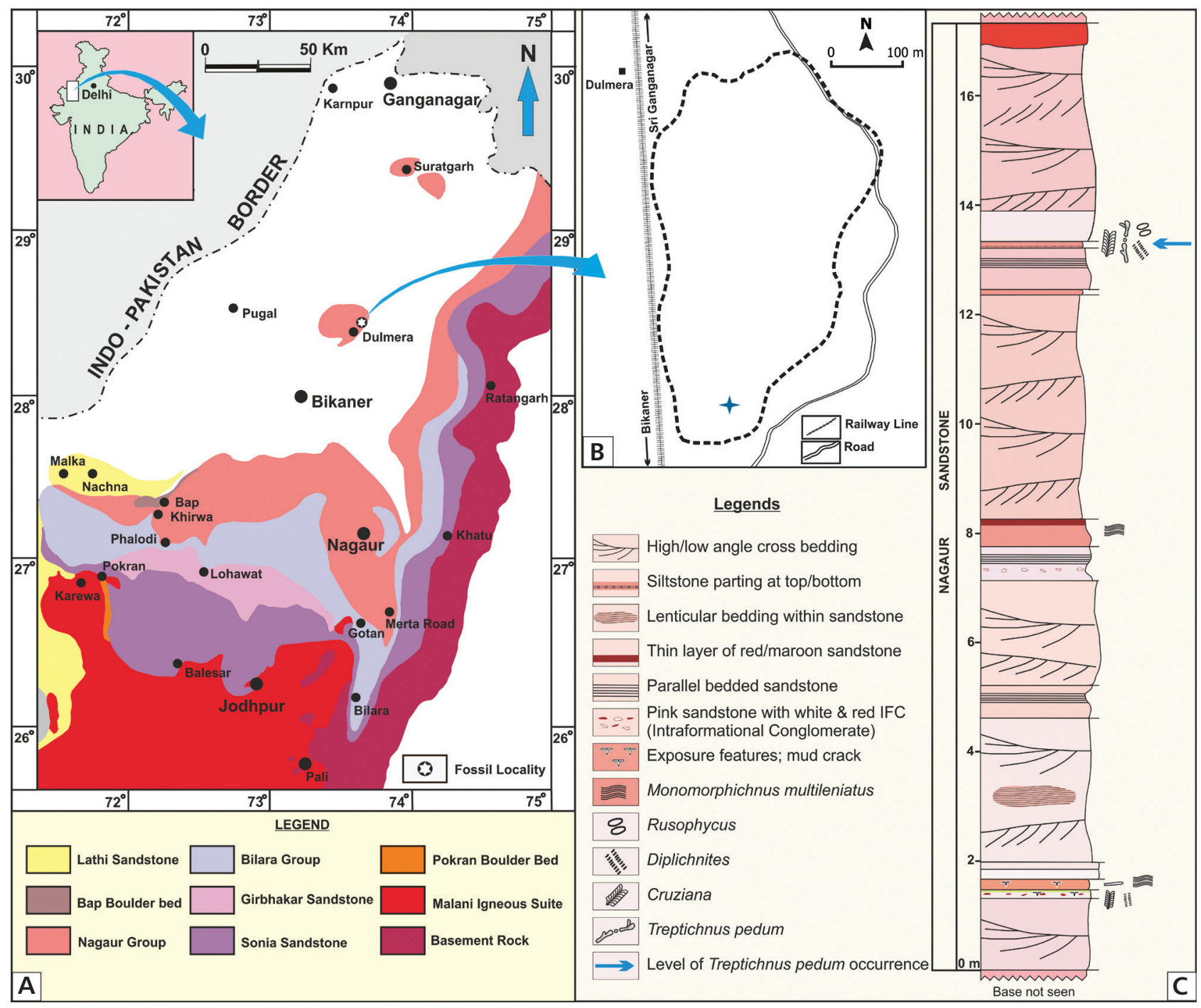

Figure 1. Geological map, area of study and litholog of the Treptichnus pedum yielding succession. $・$ A - geological map of the Marwar Supergroup, the western Rajasthan showing fossil locality exposed in Dulmera Village (after Pareek 1984). B - geographical extent of the Dulmera quarry along the Dulmera Railway Station. $\bullet \mathrm{C}$ - generalised lithology of the Nagaur Sandstone succession bearing T. pedum.

Treptichnus consists of burrows with a straight course and with segments that regularly alternate in direction (Seilacher 1955, Geyer \& Uchman 1995, Dzik, 2005). No clear morphological variations/trends are observed through its geological range (base of the Cambrian into the Early Ordovician). It is, therefore, probably correct to maintain one name - Treptichnus pedum - for such burrow structures and perhaps to distinguish the variant by informal terms (Seilacher 2007).

\section{Global distribution}

Treptichnus pedum is considered as an important fossil for demarcating the boundary between the Ediacaran and Cambrian periods (Narbonne et al. 1987, Brasier et al.
1994, Landing 1994). Concerted efforts have been made to document $T$. pedum from various successions in the world. Burrowing habit represents the infaunal behaviour of T. pedum which, for the first time, manifested in the Early Cambrian Period (Droser et al. 1999). Vannier et al. (2010) suggested that the priapulids were one of the earliest infaunal colonizers of the substrate that possibly interacted with epibenthic communities which played an important role in the early marine food chain and important sub-horizontal bioturbators in the Cambrian Substrate Revolution (Bottjer et al. 2000). Buatois et al. (2013) noted wide environmental tolerance in occurrences of $T$. pedum and supported evolutionary innovations rather than facies specific distribution. Treptichnus pedum has been recorded from all over the globe (see Table 2). 
In India, Treptichnus pedum has been reported from the Zanskar region of the Himalaya, the Lesser Himalaya, in the Mussoorie syncline (Shah \& Sudan, 1983; Singh \& Rai 1983; Parcha \& Singh 2005, 2010; Singh et al. 2014b), and the Nagaur Sandstone of the Marwar Supergroup in Rajasthan (Srivastava, 2012a, b; Pandey et al. 2014; Singh et al. 2017). Marwar specimens reported by Srivastava (2012a) are elongated, whereas, those reported by Pandey et al. (2014) have small projections.

\section{Observations on Nagaur Treptichnus pedum}

\section{Treptichnus pedum-bearing sandstone}

Ichnofossil bearing, parallel-bedded sandstone and mudstone of the Nagaur Sandstone were studied in a quarry section $\left(28^{\circ} 24.228^{\prime} \mathrm{N}, 73^{\circ} 39.514^{\prime} \mathrm{E}\right)$ Dulmera Village, close to Dulmera Railway Station $\sim 65 \mathrm{~km}$ from Bikaner District on Bikaner-Ganganagar Highway (Fig. 1B) in Rajasthan, India. A $20 \mathrm{~m}$ thick succession of the Nagaur Sandstone is exposed in the quarry faces. The sandstone is medium to coarse grained and red to maroon in colour. Some of the beds are ferruginous. Trace fossils are present in interbedded sandstone and mudstone (Fig. 2). The quarry section reveals heterolithic bedding (Fig. 1C) along with high to low angle cross-beddings (Fig. 2C), ripple marks and mudstone drapes.

Two petrographic thin sections of the ichnofossilbearing ferruginous sandstone were examined under the petrological microscope (Fig. 3A-E). The sandstone comprises mainly anhedral to subhedral quartz grains $(80 \%)$. About $40 \%$ of the quartz grains are prismatic, but most grains are rounded to sub rounded, and few are tabular and irregular in shape. Most of the prismatic quartz grains show preferred orientation (Fig. 3B, C). These grains normally show long contact with each other whereas a few grains show convex contact, and rounded and sub-rounded grains show concave contact. Diagenetic silica overgrowth, separated by fine clay between the original grain and overgrowth, has been noticed on the rounded and sub-rounded quartz grains. Monocrystalline quartz grains dominate the assemblage (95\%) with subordinate sizable population of polycrystalline grains $(5 \%)$. Square shaped opaque magnetite inclusions are noted in a few grains, while others contain inclusion of zoisite and clinozoisite. Feldspar constitutes $5 \%$ of the entire grain population. Potassium feldspars consist of tabular grains of microcline and orthoclase with characteristic cross hatched twinning, orthoclase grains, also tabular in shape, show first order grey colour. Medium-grained fresh $\mathrm{Na}$ feldspar consisting of albite shows first order grey colour and polysynthetic twinning. Lath/flaky shaped mica are constituted of biotite and muscovite showing preferred orientation similar to quartz grains. Under polarized light, biotite shows prominent pleochroism from light brown to dark brown. Muscovite shows second order interference colour under crossed polars. Most of these grains are altered at places; alteration of biotite in chlorite and $\mathrm{K} \& \mathrm{Na}$ orthoclase feldspars in kaolin has been noticed and at places kaolin is further recrystallized into sericite. Perthite grains are very rarely noticed. Quartz grains show corroded margins, which is due to clay. Iron rich (ferruginous) matrix is present in between quartz grains which are derived from alteration of biotite. Rock-fragments constitute less than 5\% which are quartzite and chert fragments which are very fine grained. Diagenetic over-growth has been noted in rounded grains. In the burrowed portion, there is complete absence of prismatic quartz grains and predominance of rounded to sub rounded quartz grains; very little feldspar is noticed, with extensive alteration product and ferruginous matter as matrix. Ferruginous matrix percentage is higher at the location of burrows, with complete absence of prismatic quartz grains and increased size sorting of quartz grains in comparison to the non fossiliferous sandstones. Iron-rich opaque minerals are considerably more common in the burrowed beds. Micaceous minerals are comparatively small in size and show preferred orientation across the burrow.

\section{Treptichnus pedum-bearing facies}

Lowermost $T$. pedum-bearing horizon is encountered $13 \mathrm{~m}$ from the base of the section exposed in the quarry. The trace fossil-bearing facies ( 2-metre-thick unit) is brown to red in colour, medium-grained sandstone with intercalations of centimetre-thick mudstone (Fig. 2). The succession can be divided into two lithofacies, namely the sandstone facies and the mudstone facies (Fig. 2A). The sandstone lithofacies is made up of decimetre-thick bands, showing cross-bedding, parallel lamination and

Figure 2. Outcrop expression of Treptichnus pedum bearing Nagaur Sandstone. • A - typical components of Treptichnus ichnofacies of Nagaur Sandstone on the quarry face at Dulmera, Rajasthan which is divisible into two lithofacies namely sandstone facies and mudstone facies. Extreme right corner depicts blow-up of mudstone facies with interspersed streaks of sand; on the left edge is shown interpretative diagram of two depositional facies representing bar and interbar; detailed sedimentary features of boxes marked as b, c, d and e are shown in respective subsequent figures. $\bullet$ B - trough cross-bedding in sandstone facies. $\cdot \mathrm{C}$ - low angle cross-bedding and trough cross-bedding. $\bullet \mathrm{D}$ - planar and undulated cross-bedded strata indicating influence of waves sandwiched between two mud dominated interbar facies. $\bullet$ E - probable tidal bundles with reactivation and bounding surfaces marked on sandstone in bar facies. 






\section{$2.0 \mathrm{~mm}$}

A
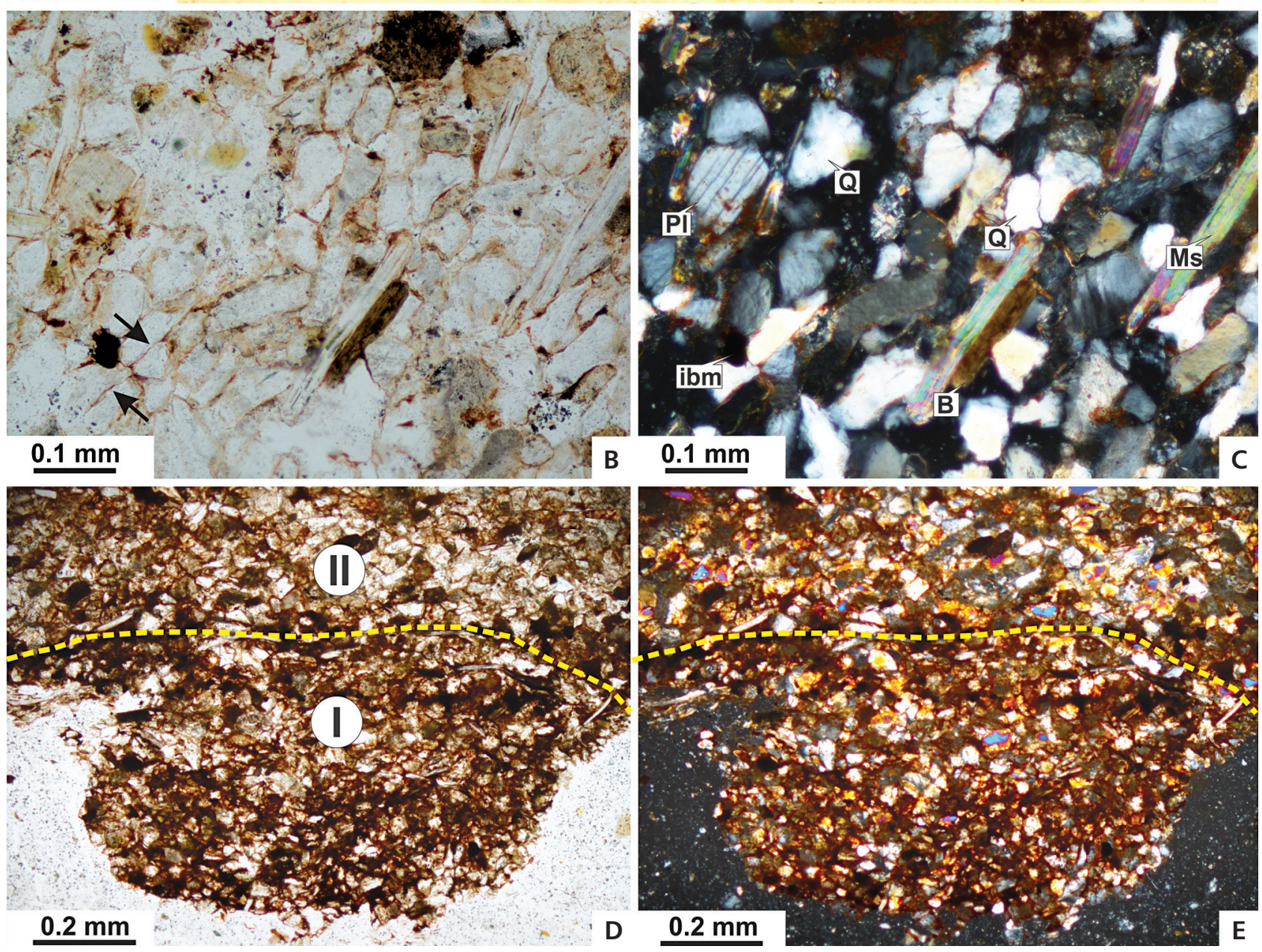

Figure 3. Petrographic thin section (slide number BSIP-16168) showing distinct host sediments and sediment trapped inside the burrow. - A - thin section of the Nagaur Sandstone under low power microscopy; black arrows show sole of the bedding plane with positive relief of burrow. $\bullet$ B - plane polarised light, black arrows indicate contact between two quartz grains. $\bullet \mathrm{C}$ - cross polarised light. Abbreviations: Q - quartz; Pl - plagioclase; Ms muscovite; ibm - iron bearing mineral; B - biotite. $\bullet \mathrm{D}$ - plane polarised light, view of burrow with host sediment. Abbreviations: I - burrow whereas; $\mathrm{II}-$ host rock. $・ \mathrm{E}-$ cross polarised light view of ' $\mathrm{D}$ '

rare small ripple cross lamination. Bedding shows trough cross-bedding, planar cross-bedding and low-angle crossbedding (Fig. 2B-D). The planar cross-bedding may show reactivation surfaces resembling tidal bundles (Fig. 2E). Some bounding surfaces in the sandstone facies are undulatory indicating influence of waves. The sandstone 


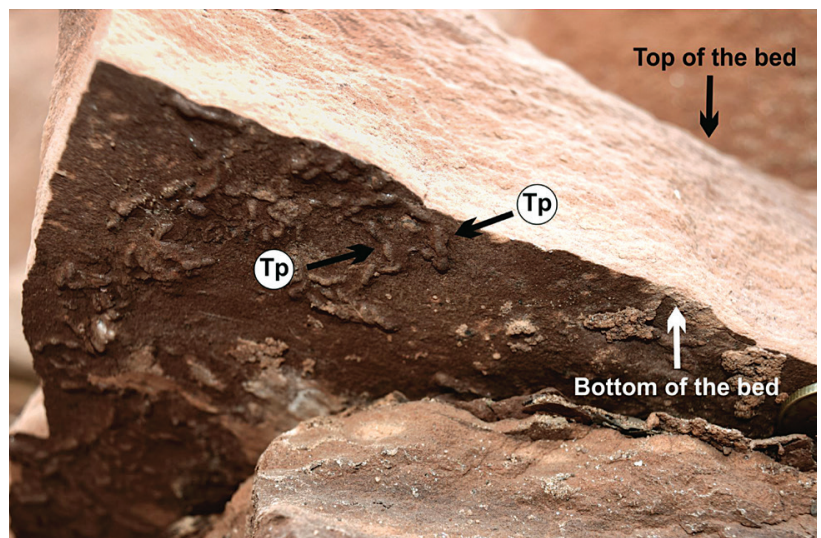

Figure 4. In-situ Treptichnus pedum noted on the sole of the fine grained sandstone, see coin for scale $=2.3 \mathrm{~cm}$.

facies suggests deposition similar to intertidal bars on sand shoal under the influence of tidal currents and wave actions (see Sharma et al. 2018). The mudstone facies is made up of a few millimetre-thick mud layers alternating with millimetre-to-centimetre thick sand layers. The mud layers show fine sandy streaks (Fig. 2A). The sand layers invariably show small ripple bedding. This lithofacies represents deposition in low-lying interbar areas protected from strong wave action and tidal currents. However, tidal processes controlled the fine sand-mud alternation. The trace fossils, Cruziana, Diplichnites, Monomorphichnus and Rusophycus along with Treptichnus pedum are preserved at the base of the sandstone layer as positive hyporelief (Fig. 4), overlying the mudstone facies. In this facies, Cruziana, Diplichnites, Rusophycus and Monomorphichnus are subordinate in abundance to T. pedum (Fig. 5). These burrows and resting traces were formed in unconsolidated sediments which constitute a softground. Above mentioned ichnotaxa, other than the T. pedum, are also reported from the horizons stratigraphically below this zone, which suggests that the documented T. pedum zone is unlikely to be the FAD. It is most likely that this assemblage mark the Stage 4 of the Series 2 of the Cambrian Period/System (Hughes 2016).

\section{Taxonomy}

Our study is based on well-preserved specimens of T. pedum recorded on the sole of seven medium-grained sandstone slabs with intercalations of mudstone. The specimens studied herein are reposited in the museum of Birbal Sahni Institute of Palaeosciences, Lucknow (collection statement number 1444).

Ichnogenus Treptichnus Miller, 1889

Ichnospecies Treptichnus pedum (Seilacher, 1955)

Figure 5A-M
Material. - Seven slabs of fine-grained sandstone consisting of 291 specimens preserved in hyporelief with fill identical to that of the slab.

Description. - Curved to slightly straight rows of short burrows. Individual burrows of equal length arranged alternating left and right at an angle; in some cases they show preferred orientation in one direction. Length of burrows varies between 1 to $17 \mathrm{~mm}($ mean $=5 \mathrm{~mm} ; \mathrm{N}=291)$; width varies between 1 to $3 \mathrm{~mm}$ (mean $=1.5 \mathrm{~mm} ; \mathrm{N}=$ 258), whereas 33 specimens show width less than $1 \mathrm{~mm}$. The gap between the two consecutive small burrows varies between $1 \mathrm{~mm}$ to $7 \mathrm{~mm}$. In some clusters, patterns of length of small burrows vary between $10 \mathrm{~mm}$ to $64 \mathrm{~mm}$ (mean $=34 \mathrm{~mm} ; \mathrm{N}=9$ ).

Remarks. - Morphometric characters of the Nagaur specimens are consistent with $T$. pedum (Seilacher, 1955). Various patterns formed by these specimens allow interpreting the behavioural activity and feeding mechanism of the makers (the priapulid worms) near the sediment-water interface. In the larger depositional basin, encompassing Salt Range, forms were described from the Neobolus Beds of Khussak Formation, Salt Range, Pakistan (Seilacher 1955).

Distribution. - Pandey et al. (2014) recorded T. pedum from the Nagaur Sandstone. Treptichnus pedum has been reported from various geological successions close to Precambrian-Cambrian boundary (Table 2) and ranges up to Ordovician (Seilacher 1969, 2007; Li 1993). Another ichnospecies of Treptichnus (e.g. T. bifurcus) has been reported up to Carboniferous (Buatois et al. 1997). Type specimen of T. pedum is from the Khussak Formation in the Salt Range (Seilacher 1955) and the stratum in which it occurs is considerably above the base of PrecambrianCambrian boundary. Here they occur just above beds that contain the brachiopod S. rugosa, early in Stage 4 (likely about $514 \mathrm{Ma}$ ) (Hughes, 2016). The age of these strata are now considered 30 million years younger than the Precambrian-Cambrian boundary (Hughes 2016). It has been shown that treptichnids, not true T. pedum, occur below the Ediacaran-Cambrian boundary (Jensen et al. 2000, 2017; Högström et al. 2013; Buatois 2018). Seilacher (2007) figured specimens of T. pedum reported from South Africa to occur in Ediacaran-Cambrian age succession but it was subsequently shown to occur in Fortunian Stage (Buatois et al. 2007, Almond et al. 2008). In different regions of Laurentia, the occurrence of T. pedum coincides with the base of the Cambrian whereas the claim of diachronism in the appearance of T. pedum in Gondwanan regions needs further investigation (Babcock et al. 2014, Buatois 2018). Available evidence on the age of the Nagaur Sandstone holding T. pedum is inconclusive. 
Table 2. Distribution list of Treptichnus pedum across the globe.

\begin{tabular}{|c|c|c|c|c|c|c|}
\hline No. & Ichnospecies & Stratigraphy & Facies & $\begin{array}{l}\text { Depositional } \\
\text { settings }\end{array}$ & Age & References \\
\hline 1. & $\begin{array}{l}\text { Treptichnus } \\
\text { pedum }\end{array}$ & $\begin{array}{l}\text { Nagaur Group, Marwar } \\
\text { Supergroup, India }\end{array}$ & $\begin{array}{l}\text { sandstone, } \\
\text { siltstone }\end{array}$ & $\begin{array}{l}\text { shallow water } \\
\text { conditions }\end{array}$ & $\begin{array}{l}\text { early } \\
\text { Cambrian }\end{array}$ & Pandey et al. (2014) \\
\hline 2. & $\begin{array}{l}\text { Treptichnus } \\
\text { pedum }\end{array}$ & $\begin{array}{l}\text { Nagaur Sandstone, Marwar } \\
\text { Supergroup, India }\end{array}$ & $\begin{array}{l}\text { sandstone, } \\
\text { siltstone }\end{array}$ & $\begin{array}{l}\text { shallow water } \\
\text { conditions }\end{array}$ & $\begin{array}{l}\text { early } \\
\text { Cambrian }\end{array}$ & Srivastava (2012a) \\
\hline 3. & $\begin{array}{l}\text { cf. Treptichnus } \\
\text { pedum }\end{array}$ & $\begin{array}{l}\text { Tal Formation, Lesser Himalaya, } \\
\text { India }\end{array}$ & sandstone & not mentioned & $\begin{array}{l}\text { early } \\
\text { Cambrian }\end{array}$ & Singh et al. (2014a) \\
\hline 4. & $\begin{array}{l}\text { Treptichnus } \\
\text { pedum }\end{array}$ & $\begin{array}{l}\text { Lolab and Tal Formation, } \\
\text { Himalaya, India }\end{array}$ & $\begin{array}{l}\text { no precise } \\
\text { data }\end{array}$ & no precise data & $\begin{array}{l}\text { early } \\
\text { Cambrian }\end{array}$ & $\begin{array}{l}\text { Shah \& Sudan (1983), } \\
\text { Singh \& Rai (1983) }\end{array}$ \\
\hline 5. & $\begin{array}{l}\text { Treptichnus } \\
\text { pedum }\end{array}$ & $\begin{array}{l}\text { Phe Formation, Zanskar region, } \\
\text { Ladakh Himalaya, India }\end{array}$ & sandstone & $\begin{array}{l}\text { shallow water } \\
\text { conditions }\end{array}$ & Cambrian & Parcha \& Singh (2010) \\
\hline 6. & $\begin{array}{l}\text { Trychophycus; } \\
\text { Phycodes }\end{array}$ & $\begin{array}{l}\text { Parahio section, Kunzum-la } \\
\text { Formation, Spiti Valley, India }\end{array}$ & $\begin{array}{l}\text { sandstone, } \\
\text { siltstone }\end{array}$ & $\begin{array}{l}\text { shallow water } \\
\text { conditions }\end{array}$ & $\begin{array}{l}\text { early } \\
\text { Cambrian }\end{array}$ & Parcha \& Singh (2005) \\
\hline 7. & $\begin{array}{l}\text { Treptichnus } \\
\text { pedum }\end{array}$ & Chapel Island Formation, Canada & $\begin{array}{l}\text { sandstone, } \\
\text { siltstone }\end{array}$ & $\begin{array}{l}\text { shallow water } \\
\text { conditions }\end{array}$ & $\begin{array}{l}\text { early } \\
\text { Cambrian }\end{array}$ & Droser et al. (2002) \\
\hline 8. & $\begin{array}{l}\text { Treptichnus } \\
\text { pedum }\end{array}$ & $\begin{array}{l}\text { Chapel Island, GSSP, Fortune } \\
\text { Head, Newfoundland, Canada }\end{array}$ & siliciclastic & not mentioned & $\begin{array}{l}\text { late } \\
\text { Ediacaran } \\
\text { to early } \\
\text { Cambrian }\end{array}$ & Gehling et al. (2001) \\
\hline 9. & $\begin{array}{l}\text { Treptichnus } \\
\text { pedum }\end{array}$ & E Newfoundland, Canada & $\begin{array}{l}\text { no precise } \\
\text { data }\end{array}$ & no precise data & $\begin{array}{l}\text { ?Furongian/? } \\
\text { Early } \\
\text { Ordovician }\end{array}$ & $\begin{array}{l}\text { Fillion \& Pickerill } \\
(1990)\end{array}$ \\
\hline 10 . & $\begin{array}{l}\text { Treptichnus } \\
\text { pedum }\end{array}$ & $\begin{array}{l}\text { Chapel Island Formation (GSSP } \\
\text { and below), Newfoundland, } \\
\text { Canada }\end{array}$ & $\begin{array}{l}\text { no precise } \\
\text { data }\end{array}$ & no precise data & $\begin{array}{l}\text { late Ediacaran } \\
\text { and early } \\
\text { Cambrian }\end{array}$ & $\begin{array}{l}\text { Crimes \& Anderson } \\
\text { (1985), Narbonne } \\
\text { et al. (1987), Brasier } \\
\text { et al. (1994), Landing } \\
\text { (1994) }\end{array}$ \\
\hline 11. & $\begin{array}{l}\text { Treptichnus } \\
\text { pedum }\end{array}$ & $\begin{array}{l}\text { Random Formation, SE } \\
\text { Newfoundland, Canada }\end{array}$ & $\begin{array}{l}\text { no precise } \\
\text { data }\end{array}$ & no precise data & $\begin{array}{l}\text { early } \\
\text { Cambrian }\end{array}$ & Narbonne et al. (1987) \\
\hline 12. & $\begin{array}{l}\text { Treptichnus } \\
\text { pedum }\end{array}$ & $\begin{array}{l}\text { Boya Formation, Cassiar } \\
\text { Mountain, Canada }\end{array}$ & $\begin{array}{l}\text { no precise } \\
\text { data }\end{array}$ & no precise data & $\begin{array}{l}\text { early } \\
\text { Cambrian }\end{array}$ & $\begin{array}{l}\text { Fritz 1980, Fritz et al. } \\
\text { (1983), Droser et al. } \\
(1999)\end{array}$ \\
\hline 13. & $\begin{array}{l}\text { Treptichnus } \\
\text { pedum }\end{array}$ & $\begin{array}{l}\text { Lower Vampire Formation, } \\
\text { Wernecke Mountains, Canada }\end{array}$ & $\begin{array}{l}\text { no precise } \\
\text { data }\end{array}$ & no precise data & $\begin{array}{l}\text { early } \\
\text { Cambrian }\end{array}$ & $\begin{array}{l}\text { Nowlan et al. (1985), } \\
\text { Droser et al. (1999) }\end{array}$ \\
\hline 14. & $\begin{array}{l}\text { Treptichnus } \\
\text { pedum }\end{array}$ & $\begin{array}{l}\text { Ingta Formation; Backbone } \\
\text { Ranges Formation; Vampire } \\
\text { Formation, Mackenzie Mountain, } \\
\text { NW Canada }\end{array}$ & $\begin{array}{l}\text { no precise } \\
\text { data }\end{array}$ & no precise data & $\begin{array}{l}\text { late } \\
\text { Ediacaran } \\
\text { and early } \\
\text { Cambrian }\end{array}$ & $\begin{array}{l}\text { MacNaughton \& } \\
\text { Narbonne (1999) }\end{array}$ \\
\hline 15. & $\begin{array}{l}\text { cf. Treptichnus } \\
\text { pedum }\end{array}$ & Guachos Formation, Argentina & sandstone & $\begin{array}{l}\text { shallow water } \\
\text { conditions }\end{array}$ & $\begin{array}{l}\text { early } \\
\text { Cambrian }\end{array}$ & Seilacher et al. (2005) \\
\hline 16. & $\begin{array}{l}\text { Treptichnus } \\
\text { pedum }\end{array}$ & $\begin{array}{l}\text { Balcare Formation, Buenos Aires } \\
\text { Province, Argentina }\end{array}$ & $\begin{array}{l}\text { no precise } \\
\text { data }\end{array}$ & no precise data & Cambrian & $\begin{array}{l}\text { Regalia \& Herrera } \\
\text { (1981) }\end{array}$ \\
\hline 17. & $\begin{array}{l}\text { Treptichnus } \\
\text { pedum }\end{array}$ & Uratanna Formation, S Australia & $\begin{array}{l}\text { sandstone, } \\
\text { siltstone }\end{array}$ & $\begin{array}{l}\text { shallow water } \\
\text { conditions }\end{array}$ & $\begin{array}{l}\text { early } \\
\text { Cambrian }\end{array}$ & Droser et al. (1999) \\
\hline 18. & $\begin{array}{l}\text { Treptichnus } \\
\text { pedum }\end{array}$ & $\begin{array}{l}\text { Parachilna Formation, Flinders } \\
\text { Range, Australia }\end{array}$ & $\begin{array}{l}\text { no precise } \\
\text { data }\end{array}$ & no precise data & $\begin{array}{l}\text { early } \\
\text { Cambrian }\end{array}$ & Daily (1972) \\
\hline 19. & $\begin{array}{l}\text { Treptichnus } \\
\text { pedum }\end{array}$ & $\begin{array}{l}\text { Arumbera Formation, Dinkey } \\
\text { Creek Beds, Amadeus Basin, } \\
\text { Australia }\end{array}$ & $\begin{array}{l}\text { no precise } \\
\text { data }\end{array}$ & no precise data & $\begin{array}{l}\text { early } \\
\text { Cambrian }\end{array}$ & $\begin{array}{l}\text { Glaessner (1969), } \\
\text { Daily (1972), Walter } \\
\text { et al. }(1989)\end{array}$ \\
\hline 20. & $\begin{array}{l}\text { Treptichnus } \\
\text { pedum }\end{array}$ & Urusis Formation, S Namibia & sandstone & not mentioned & $\begin{array}{l}\text { early } \\
\text { Cambrian }\end{array}$ & $\begin{array}{l}\text { Jensen \& Runnegar } \\
(2005)\end{array}$ \\
\hline 21. & $\begin{array}{l}\text { Treptichnus } \\
\text { pedum }\end{array}$ & $\begin{array}{l}\text { Upper Nomtsas Formation, } \\
\text { Spitskopf Member and Urusis } \\
\text { Formation of Nama Group, } \\
\text { Namibia }\end{array}$ & sandstone & $\begin{array}{l}\text { shallow water } \\
\text { conditions }\end{array}$ & $\begin{array}{l}\text { early } \\
\text { Cambrian }\end{array}$ & Wilson et al. (2012) \\
\hline
\end{tabular}


Table 2. continued.

\begin{tabular}{|c|c|c|c|c|c|c|}
\hline No. & Ichnospecies & Stratigraphy & Facies & $\begin{array}{l}\text { Depositional } \\
\text { settings }\end{array}$ & Age & References \\
\hline 22. & $\begin{array}{l}\text { Treptichnus } \\
\text { pedum }\end{array}$ & Nama Group, Namibia & $\begin{array}{l}\text { sandstone, } \\
\text { siltstone }\end{array}$ & not mentioned & $\begin{array}{l}\text { early } \\
\text { Cambrian }\end{array}$ & Jensen et al. (2000) \\
\hline 23. & $\begin{array}{l}\text { Treptichnus } \\
\text { pedum }\end{array}$ & $\begin{array}{l}\text { Gross Aub Formation and } \\
\text { Nomtas Formation, South } \\
\text { Namibia }\end{array}$ & $\begin{array}{l}\text { no precise } \\
\text { data }\end{array}$ & no precise data & $\begin{array}{l}\text { early } \\
\text { Cambrian }\end{array}$ & $\begin{array}{l}\text { Germs (1972), Crimes } \\
\text { \& Germs (1982), } \\
\text { Geyer \& Uchman } \\
(1995)\end{array}$ \\
\hline 24. & $\begin{array}{l}\text { Treptichnus } \\
\text { pedum }\end{array}$ & Death Valley, USA & $\begin{array}{l}\text { sandstone, } \\
\text { siltstone }\end{array}$ & not mentioned & $\begin{array}{l}\text { early } \\
\text { Cambrian }\end{array}$ & $\begin{array}{l}\text { Corsetti \& Hagadorn } \\
(2000)\end{array}$ \\
\hline 25. & $\begin{array}{l}\text { Treptichnus } \\
\text { pedum }\end{array}$ & $\begin{array}{l}\text { Bright Angel Shale, Grand } \\
\text { Canyon, USA }\end{array}$ & $\begin{array}{l}\text { no precise } \\
\text { data }\end{array}$ & no precise data & $\begin{array}{l}\text { early to } \\
\text { middle } \\
\text { Cambrian }\end{array}$ & $\begin{array}{l}\text { Seilacher (1956), } \\
\text { Eliot \& Martin (1987) }\end{array}$ \\
\hline 26. & $\begin{array}{l}\text { Treptichnus } \\
\text { pedum }\end{array}$ & $\begin{array}{l}\text { Deep Spring Formation, Campito } \\
\text { Formation, White Mountains, } \\
\text { USA }\end{array}$ & $\begin{array}{l}\text { no precise } \\
\text { data }\end{array}$ & no precise data & $\begin{array}{l}\text { early } \\
\text { Cambrian }\end{array}$ & Alpert (1977) \\
\hline 27. & $\begin{array}{l}\text { Treptichnus } \\
\text { pedum }\end{array}$ & $\begin{array}{l}\text { Gongwusu Formation, Inner } \\
\text { Mangolia, China }\end{array}$ & $\begin{array}{l}\text { no precise } \\
\text { data }\end{array}$ & no precise data & $\begin{array}{l}\text { Middle } \\
\text { Ordovician }\end{array}$ & $\operatorname{Li}(1993)$ \\
\hline 28. & $\begin{array}{l}\text { Treptichnus } \\
\text { pedum }\end{array}$ & $\begin{array}{l}\text { Kaili Formation, Guizhou } \\
\text { Province (S China) }\end{array}$ & $\begin{array}{l}\text { no precise } \\
\text { data }\end{array}$ & no precise data & $\begin{array}{l}\text { middle } \\
\text { Cambrian }\end{array}$ & $\begin{array}{l}\text { Yang (1994), } \\
\text { Wang \& Wang (2006) }\end{array}$ \\
\hline 29. & $\begin{array}{l}\text { Treptichnus } \\
\text { pedum }\end{array}$ & $\begin{array}{l}\text { Yu'anshan Formation, Yunnan } \\
\text { Province, South China }\end{array}$ & $\begin{array}{l}\text { no precise } \\
\text { data }\end{array}$ & no precise data & $\begin{array}{l}\text { early } \\
\text { Cambrian }\end{array}$ & Zhu (1997) \\
\hline 30. & $\begin{array}{l}\text { Treptichnus } \\
\text { pedum }\end{array}$ & $\begin{array}{l}\text { Wisniowka Formation, Holy } \\
\text { Cross Mountains, Poland }\end{array}$ & $\begin{array}{l}\text { no precise } \\
\text { data }\end{array}$ & no precise data & Furongian & $\begin{array}{l}\text { Orlowski \& Żylińska } \\
\text { (1996) }\end{array}$ \\
\hline 31. & $\begin{array}{l}\text { Treptichnus } \\
\text { pedum }\end{array}$ & $\begin{array}{l}\text { Ocieseki Formation, Holy Cross } \\
\text { Mountains, Poland }\end{array}$ & $\begin{array}{l}\text { no precise } \\
\text { data }\end{array}$ & no precise data & $\begin{array}{l}\text { early } \\
\text { Cambrian }\end{array}$ & Orlowski (1989) \\
\hline 32. & $\begin{array}{l}\text { Treptichnus } \\
\text { pedum }\end{array}$ & Platysolenites Zone, SE Poland & $\begin{array}{l}\text { no precise } \\
\text { data }\end{array}$ & no precise data & $\begin{array}{l}\text { early } \\
\text { Cambrian }\end{array}$ & Paczesna $(1985,1986)$ \\
\hline 33. & $\begin{array}{l}\text { Treptichnus } \\
\text { pedum }\end{array}$ & $\begin{array}{l}\text { Detrital Beds, Sierra De } \\
\text { Guadelupe, Spain }\end{array}$ & $\begin{array}{l}\text { no precise } \\
\text { data }\end{array}$ & no precise data & $\begin{array}{l}\text { early } \\
\text { Cambrian }\end{array}$ & Liñán (1984) \\
\hline 34. & $\begin{array}{l}\text { Treptichnus } \\
\text { pedum }\end{array}$ & $\begin{array}{l}\text { Vegadeo Limestone, Herreria } \\
\text { Sandstone, Cantabrian Mountains, } \\
\text { N Spain }\end{array}$ & sandstone & no precise data & $\begin{array}{l}\text { early } \\
\text { Cambrian }\end{array}$ & $\begin{array}{l}\text { Crimes et al. }(1977), \\
\text { Baldwin (1977), } \\
\text { Legg (1985) }\end{array}$ \\
\hline 35. & $\begin{array}{l}\text { Treptichnus } \\
\text { pedum }\end{array}$ & $\begin{array}{l}\text { Mickwitzia Sandstone, South } \\
\text { Central Sweden }\end{array}$ & $\begin{array}{l}\text { no precise } \\
\text { data }\end{array}$ & no precise data & $\begin{array}{l}\text { early } \\
\text { Cambrian }\end{array}$ & Jensen (1997) \\
\hline 36. & $\begin{array}{l}\text { Treptichnus } \\
\text { pedum }\end{array}$ & $\begin{array}{l}\text { Tornetrask Formation, } \\
\text { Dividalen Group, N Sweden }\end{array}$ & $\begin{array}{l}\text { no precise } \\
\text { data }\end{array}$ & no precise data & $\begin{array}{l}\text { late } \\
\text { Ediacaran } \\
\text { and early } \\
\text { Cambrian }\end{array}$ & Jensen \& Grant (1998) \\
\hline 37. & $\begin{array}{l}\text { Treptichnus } \\
\text { pedum }\end{array}$ & $\begin{array}{l}\text { Klipbak Formation, Vanrhynsdrop } \\
\text { Group, South Africa }\end{array}$ & sandstone & $\begin{array}{l}\text { shallow marine } \\
\text { clastic setting }\end{array}$ & $\begin{array}{l}\text { early } \\
\text { Cambrian }\end{array}$ & Buatois et al. (2013) \\
\hline 38. & $\begin{array}{l}\text { Treptichnus } \\
\text { pedum }\end{array}$ & $\begin{array}{l}\text { Neobolus beds, Salt Range, } \\
\text { Pakistan }\end{array}$ & $\begin{array}{l}\text { no precise } \\
\text { data }\end{array}$ & no precise data & $\begin{array}{l}\text { early } \\
\text { Cambrian }\end{array}$ & Seilacher (1955) \\
\hline 39. & $\begin{array}{l}\text { Treptichnus } \\
\text { pedum }\end{array}$ & $\begin{array}{l}\text { Puerto Blanco Formation, Sonora, } \\
\text { Mexico }\end{array}$ & sandstone & not mentioned & $\begin{array}{l}\text { early } \\
\text { Cambrian }\end{array}$ & Sour-Tovar et al. (2007) \\
\hline 40. & $\begin{array}{l}\text { Treptichnus } \\
\text { pedum }\end{array}$ & $\begin{array}{l}\text { Melez Chorgrane Formation, } \\
\text { Libya }\end{array}$ & $\begin{array}{l}\text { no precise } \\
\text { data }\end{array}$ & no precise data & $\begin{array}{l}\text { Early } \\
\text { Ordovician }\end{array}$ & Seilacher (1969) \\
\hline 41. & $\begin{array}{l}\text { Treptichnus } \\
\text { pedum }\end{array}$ & Rovno Formation, Ukraine & $\begin{array}{l}\text { no precise } \\
\text { data }\end{array}$ & no precise data & $\begin{array}{l}\text { early } \\
\text { Cambrian }\end{array}$ & $\begin{array}{l}\text { Fedonkin (1983), } \\
\text { Palij (1976) }\end{array}$ \\
\hline 42. & $\begin{array}{l}\text { Treptichnus } \\
\text { pedum }\end{array}$ & $\begin{array}{l}\text { Breivik Formation, Finnmark, } \\
\text { Norway }\end{array}$ & $\begin{array}{l}\text { no precise } \\
\text { data }\end{array}$ & no precise data & $\begin{array}{l}\text { early } \\
\text { Cambrian }\end{array}$ & $\begin{array}{l}\text { Banks (1970), Føyn \& } \\
\text { Glaessner (1979) }\end{array}$ \\
\hline 43. & $\begin{array}{l}\text { Treptichnus } \\
\text { pedum }\end{array}$ & Lontova Formation, Estonia & $\begin{array}{l}\text { no precise } \\
\text { data }\end{array}$ & no precise data & $\begin{array}{l}\text { early } \\
\text { Cambrian }\end{array}$ & $\begin{array}{l}\text { Palij (1976), } \\
\text { Palij et al. (1983) }\end{array}$ \\
\hline 44. & $\begin{array}{l}\text { Treptichnus } \\
\text { pedum }\end{array}$ & Buen Formation, N Greenland & $\begin{array}{l}\text { no precise } \\
\text { data }\end{array}$ & no precise data & $\begin{array}{l}\text { early } \\
\text { Cambrian }\end{array}$ & Bryant \& Pickerill (1990) \\
\hline
\end{tabular}


Table 3. Pearson correlation coefficients (r) of Treptichnus pedum dimensions recorded from the Nagaur Group. Abbreviations: $\mathrm{N}$ - number of specimens; ${ }^{*}$ - statistically significant correlation at the 0.01 level (2-tailed).

\begin{tabular}{llccc}
\hline & & Length & Width & Gap \\
\hline Length & r & 1 & $0.407^{* *}$ & -0.051 \\
& Sig. (2-tailed) & & 0.000 & 0.488 \\
& $\mathrm{~N}$ & 291 & 258 & 190 \\
\hline Width & r & $0.407^{* *}$ & 1 & -0.125 \\
& Sig. (2-tailed) & 0.000 & & 0.112 \\
& N & 258 & 258 & 163 \\
\hline Gap & r & -0.051 & -0.125 & 1 \\
& Sig. (2-tailed) & 0.488 & 0.112 & \\
& N & 190 & 163 & 190 \\
\hline
\end{tabular}

\section{Statistical analysis}

In the present study, three components of each burrow, i.e. length, width and consecutive gaps between segments of $T$. pedum have been measured in order to explore the variation of these parameters at the ichnogeneric or ichnospecies level. Statistical analyses including Pearson product-moment correlation, box-plot and hierarchical cluster analysis were performed to assess the variability. The techniques of cluster analysis are useful tools for data analysis in various situations. These techniques are commonly used to search for natural groupings in the objects based on certain variables so that similar objects are in the same cluster or group. In some situations, cluster analysis methods can also be used to produce groups that form the basis of classification. Cluster analysis can be used for predictive purpose to determine the group based on certain variables. There are various algorithms available for cluster analysis (Everitt 1978). In this case we used Ward's minimum variance algorithm which is most appropriate for this data set and produces a dendrogram that is a pictoral representation of relationships (see Figs 6, 7). A total of 291 specimens, present on seven slabs, have been counted where length of burrows varies between 1 to $17 \mathrm{~mm}$ (median $=4 \mathrm{~mm}$, $\mathrm{N}=291$ ); width varies between $<1$ to $3 \mathrm{~mm}$ (median $=$ $1 \mathrm{~mm}, \mathrm{~N}=291$ ). The gap between two segments ranges $<1$ to $7 \mathrm{~mm}$ with a mean of $1 \mathrm{~mm}$. Pearson product-moment correlation suggest that there was a significant positive correlation between length and width (Table 3). As the length increases, the width of each segment also increases, but no significant correlation between the length and gap as well as between the width and gap was observed. Similarly, the box-plot for the length was calculated, the median range quartile was found between of 3 to 5 while the upper quartile range was observed at approximately 7. There were few outliers, in length in the specimens $181,182,242,199,112,100$. Similarly, for the width and the gap, the box-plot showed varying outliers viz. 119, $215,118,247,268,280$ and inliers were 278, 276, 275, 277 , while in the gap the outliers were $101,35,150,189$, $149,190,100$ and 36 . These outliers are calculated if the specimen characteristics (length, width or gap) falls outside mean \pm 3 (standard deviation) bands. Box-plots do not show any significant relationship of the gap with the length and width. The hierarchical cluster analysis was performed on the length, width and gap and it was found that all the specimens belong to one species. However, for the specimen 182, the result varied and this may possibly be due to the outlier, hence, it can be omitted (Fig. 8A-F). When all the outliers were removed, all the specimens were grouped into two: specimen numbers $35,183,145,146,182$, $98,99,157$ fall in one group and rest of the 282 specimens in the other group. Thus, it is established in the firm context that all the specimens are from one major group.

\section{Discussion}

\section{Palaeoecology}

Trace fossils reflect the behaviour of animals responsible for their formation (Seilacher 1967), but the record is biased towards activities of infaunal organisms, and their distribution is strongly controlled by environmental factors (Buatois \& Mángano 2011). Treptichnus pedum, Rusophycus isp., Cruziana isp. and Diplichnites isp. are important trace fossils in the Nagaur assemblage to ascertain the age and palaeoecology (Fig. 9A-F). A number of scratch marks and burrows, supposed to be produced by different types of trilobites or other arthropods, are reported from this unit (Kumar \& Pandey 2008, 2010; Sharma \& Pandey 2011; Singh et al. 2013; Ahmad \& Kumar 2014).

Treptichnus pedum is characteristically found in shallow, fully marine to marginal-marine environments

Figure 5. Distribution pattern and characteristic features of Treptichnus pedum recovered from Nagaur Sandstone, Dulmera quarry. $\bullet$ A - small segments of burrow consecutively connected which depicts the movement of the organism, BSIP-41046. $\bullet$ B - the interspaces between the two consecutive burrow segments indicate sediment-water interface zone where the animal was out of sediment cover, BSIP-41046. $\bullet$ C, D, E - multiple burrows are attached to main master burrow, BSIP-41043. • F, H - linear arrangement of burrow system, BSIP-41045. • G - randomly distributed burrow geometry, BSIP-41045. - I - in hyporelief, T. pedum overlapping the Cruziana isp., BSIP-41045. • J - little gap between the two consecutive burrows, BSIP-41045. $・ \mathrm{~K}$ - another pattern of T. pedum burrow, BSIP-41043. • L, M - overlapping of T. pedum and Cruziana, BSIP-41045. Scale bars: A-E, $\mathrm{K}-\mathrm{M}=0.5 \mathrm{~cm} ; \mathrm{F}-\mathrm{J}=1 \mathrm{~cm}$. 




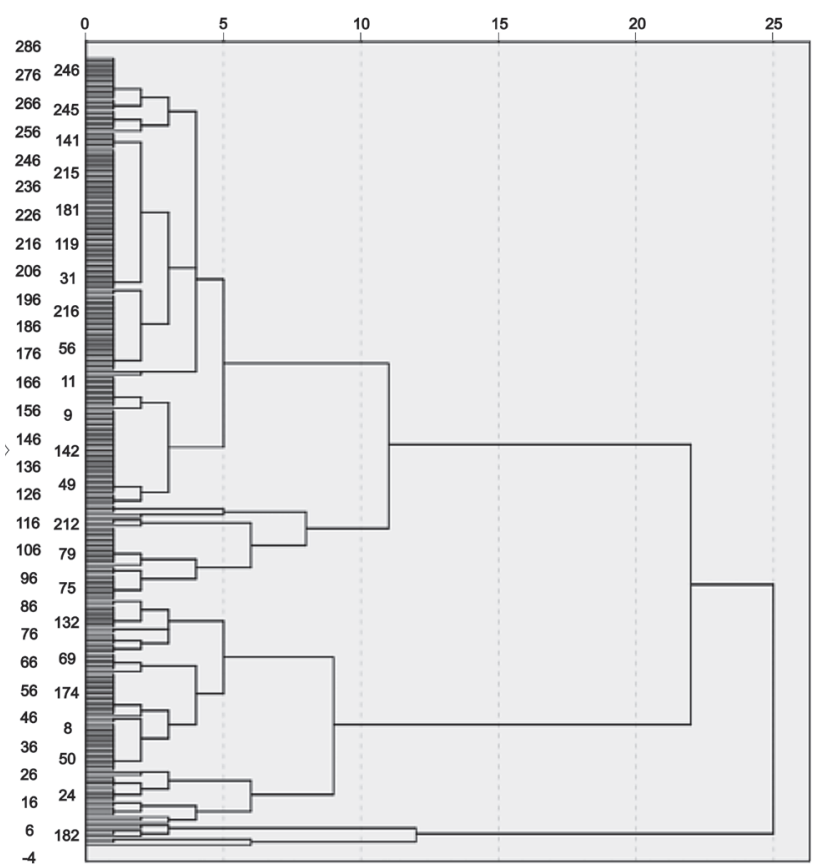

Figure 6. Dendrogram using Average Linkage algorithm (between groups) of Treptichnus pedum $(\mathrm{N}=291)$, showing all the burrows were formed by single species (Priapulid). The vertical axis gives the specimen number while horizontal axis gives the distance which is a measure of closeness of clusters.

(Geyer \& Uchman 1995, MacNaugton \& Narbonne 1999, Mángano et al. 2012). Rusophycus and Cruziana may occur in brackish-water environment (Mángano \& Buatois 2003). Rusophycus is a typical resting form of the trilobite (Buatois \& Mángano 2011; for general review see Mángano et al. 2012). Cruziana is commonly preserved as the convex hyporelief cast of the troughshaped burrow, rather than original concave burrow. Most of Cruziana are interpreted to be crawling traces of trilobites/other arthropods, since these bear scratch traces of stiff legs as they tunnelled through the shallow sediment. With high ichno-diversity the Nagaur assemblage is dominated by horizontal traces which are formed by the ichnofauna characteristic of low energy conditions (Buatois \& Mángano 2011) typically included in the Cruziana ichnofacies. Although trace fossils in the softground unit of the Nagaur Sandstone are abundant, no body fossil has been documented, such situation is rather common to note in other successions elsewhere. Burrows by animals are formed to address the four life cycle related issues: respiration, feeding, reproduction, and protection (Bromley 1990, 1996; Mángano \& Buatois 1999). Life of the organisms within the substrate is insulated from environmental and biological stress (salinity fluctuations, erosion, desiccation and predation). As mentioned above, the Nagaur assemblage is preserved in sandstonemudstone bar-interbar facies. No direct evidence is avail-

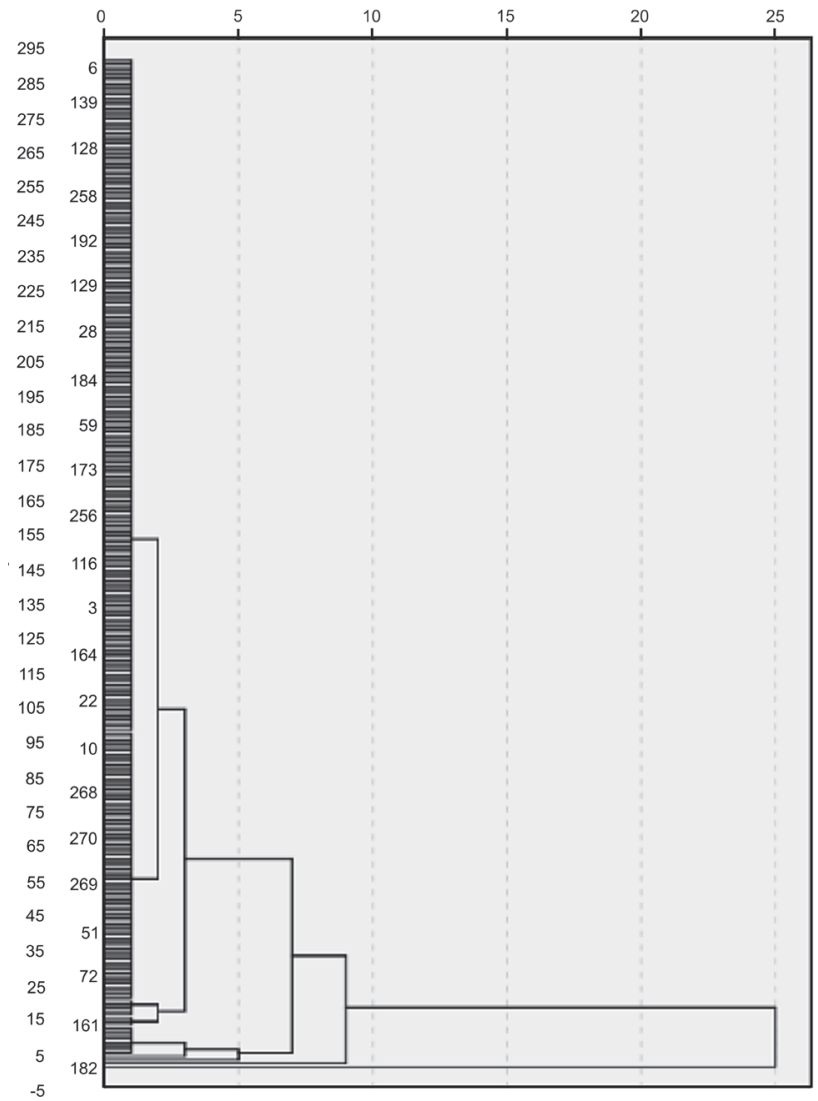

Figure 7. Dendrogram using Average Linkage algorithm (between groups) of Treptichnus pedum $(\mathrm{N}=291)$ shows that all specimen comes under 1 cluster except specimen no. 182 which could be an outlier. The vertical axis gives the specimen number while horizontal axis gives the distance which is a measure of closeness of clusters.

able for the reason to burrow the softground but possibly these burrows were formed in search of food.

\section{Palaeobiology}

Priapulids live under the thin film of sediments and sometimes, they comes to the surface or near the sedimentwater interface for oxygen and nutrient; for managing so, they propel themselves in upward curving projection that breaches into the sediment surface (Seilacher 1955, Seilacher \& Hemleben 1966, Vannier et al. 2010). Seilacher (1955), Jensen (1997) and Dzik (2005) considered that treptichnid burrows were mainly produced during feeding near the sediment-water interface but also intended for protection and shelter. Another important feature that adds to burrow complexity burrow is the pattern of outline morphology, i.e. straight to curved, locally discontinuous in a linear fashion. There is the main tunnel from which many small buds like projections are attached. These small bud-like projections are arranged 


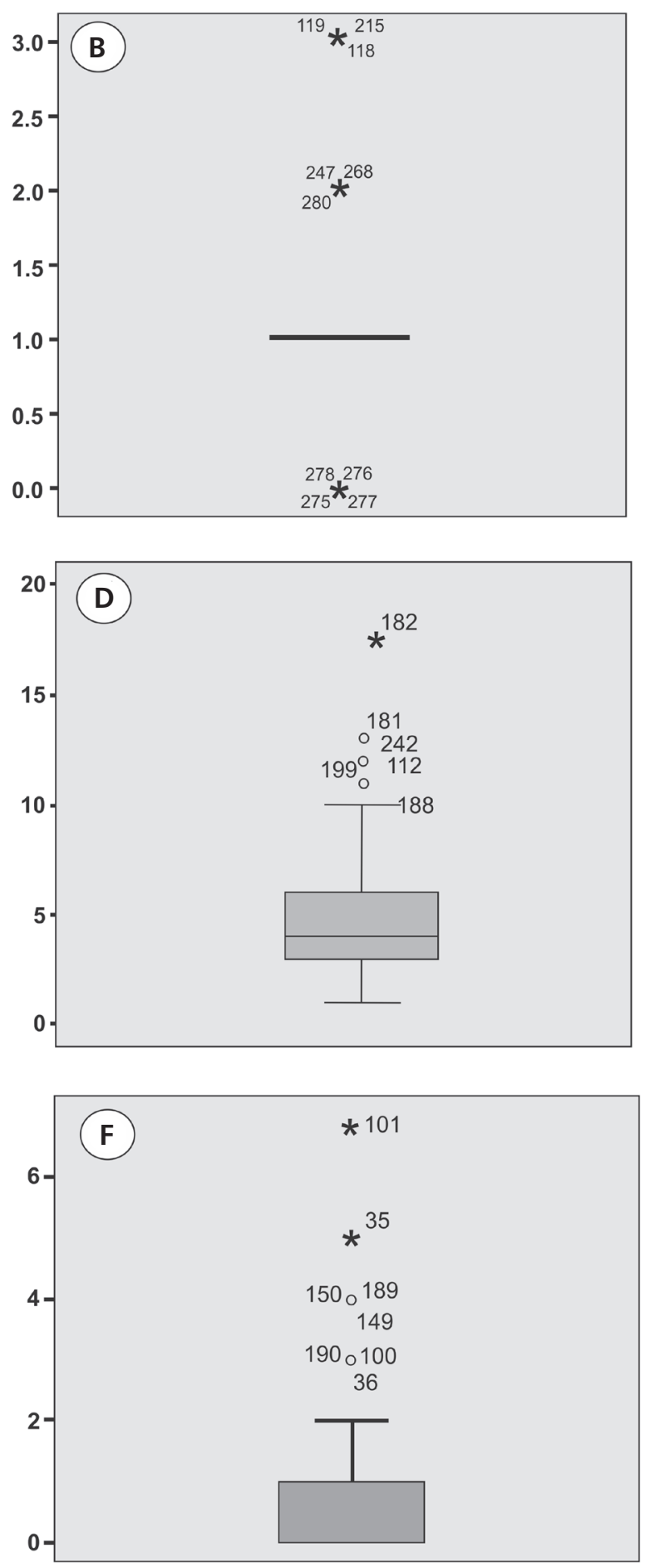

Figure 8. A-F - results of the Hierarchical Cluster Analysis performed using three parameters, i.e. Width, Length and Gap of Treptichnus pedum. Figures B, D and F represents box-plot which displays the distribution of data based on the five number summaries: minimum, first quartile, median, third quartile, and maximum. In the box plot the rectangle spans the first quartile to the third quartile with in between and bars above and below the box show the locations of the minimum and maximum. Above and below the minimum and maximum it displays the observations which are outliers (observations which are above and below mean $\pm 3 \times$ Standard deviation). 
on one side or, in some cases, they occur on both sides of the main tunnel. These projections signify the behavioural movement of the animal. Wilson et al. (2012) suggested the functional biology of priapulids in two ways: a) the animal might have lived infaunally to avoid predation or desiccation, and appeared on the surface episodically to feed and receive oxygen; and b) the animal might have been a deposit feeder that surfaced regularly to exchange gases and perhaps to disperse eggs, sperm or fertilized eggs. The latter appears more plausible because if the priapulid were able to find food on the surface then there is no valid reason for them to build three-dimensional burrows, although the safety from other predators could be the reason in that case the burrow system reflects the escape mechanism from predation.

\section{Taphonomy}

In the present study, a model is proposed to elaborate the taphonomical aspects of the Nagaur specimens, including burrowing (Fig. 3D, E) and movement of T. pedum (Fig. $10 \mathrm{~A}-\mathrm{F})$. As stated earlier, there were gaps between the two segments which might have been formed during the time when the animal was out of the sediment layer (Fig. $10 \mathrm{D}, \mathrm{F})$. The length of the individual segments also varies as some segments extend up to centimetres scale, while a few hardly reach the millimetre-scale. It is presumed that when the animal moved into softer sediments it made long individual segments (see Srivastava 2012a, fig. 3f) and on the contrary when the sediments were relatively consolidated and hard, the resultant segments were small (Fig. 5D). The purpose of multiple exits noted in the burrow system still requires a suitable explanation as in an open tunnel system active ventilation would have been easier in a U-shaped structure with only two openings to the surface. Most likely, the multiple exits served the role of passive ventilation and/or for trapping small biotic elements that drifted inside the burrow from the sedimentwater interface. Alternatively, they could have become actively backfilled upon completion of the next exit. Treptichnus pedum is prominently more three dimensional than the burrow of any other typical under mat miners.

The appearance of T. pedum in the Nagaur Sandstone of the Marwar Supergroup symbolises the change in the ecology and depositional realm and also shows the evolutionary trend in the early metazoan biosphere. The ichnogenera Rusophycus, Diplichnites and Cruziana are also found preserved on the same surface however, no short range taxon is found in the assemblage (Fig. 9E) suggesting that the zones of T. pedum cross over in these strata and occur together with Rusophycus throughout the Cambrian (Jensen \& Mens 2001, Hofmann et al. 2012, Srivastava 2012a, Pandey et al. 2014). The Agronomic Revolution (Seilacher \& Pflüger 1994, Seilacher 1999) subsequently triggered another revolution in selection of the substrate changes by the benthic organisms (Mángano $\&$ Buatois 2017). The radiation of burrowing metazoan in the early Phanerozoic is considered to involve the evolution of hardgrounds and complex organismsubstrate interactions, also known as 'Cambrian Substrate Revolution' (Bottjer et al. 2000, Tarhan \& Droser 2014). The lack of or hunt for nutrition on the substrate compelled the organisms, building simple burrows, to intrude deeper into the sediment crafting a complex burrow system. Treptichnus pedum accounts for the first infaunal activity in the Nagaur Sandstone of the Marwar Basin. The ichnospecies Bergaueria cf. perata occasionally found associated with T. pedum is another example of vertical burrowing. Though, the ichnospecies $T$. pedum is also reported from the various depositional environments (Buatois et al. 2013) but mostly it represents shallow marine setting; in the case of the Nagaur Group, the sedimentary structures of sandstone and mudstone facies suggest that the Nagaur Sandstone represents deposition in low-lying interbar areas protected from strong wave action and tidal currents indicating shallow marine setting. Penetration depths are $1-5 \mathrm{~mm}$ which indicates that the animal occupied shallow tiers. It also suggests that the sufficient nutrient saturation was available immediately below the substrate and there was no need to dig deeper burrows by the organisms. The statistical analysis also concludes that all these small projections were formed by the same priapulid as all of them falls in same hierarchy in the hierarchical cluster analysis (dendrogram) and also the box-plot analysis, which shows that the peculiar pattern of burrowing might be the end result of their behavioural activity. The gaps in between the two consecutive projections infer that the animal lived near the sediment-water interface and represents the time frame when the animal was out of the sediment.

\section{Implications on Precambrian-Cambrian boundary}

In many places, specifically Namibia and Spain, treptichnids appeared below the Ediacaran-Cambrian

Figure 9. Other trace fossils in the assemblage recorded from the Nagaur Sandstone (BSIP specimen no. BSIP-41046). • A-D - Rusophycus isp. preserved as a negative hyporelief. $\bullet \mathrm{E}$ - preservation of Rusophycus isp. and Diplichnites isp. on the same bedding. $\bullet \mathrm{F}-$ Diplichnites isp. preserved on the Nagaur Sandstone. Scale bars: $0.5 \mathrm{~cm}$ (white bar) and $1 \mathrm{~cm}$ (black bar). 





Figure 10. Cartoons illustrating the suggested stages of formation and preservation of burrows of Treptichnus pedum reported from the Nagaur Sandstone. - A - block diagram showing the level of preservation of simple to complex burrow system and other miscellaneous trace fossils noted at 13 metres level of Nagaur Sandstone. Abbreviations: Tp Treptichnus pedum; $\mathrm{Br}$ - Bergaueria; $\mathrm{Cr}$ - Cruziana; $\mathrm{Rs}$ - Rusophycus; $\mathrm{Dp}$-Diplichnites. $\cdot \mathrm{B}$ - mould of T. pedum burrow as it would appear on the sole of the sandstone bed (three-dimensional morphology of burrow after cleaning and partitioning from mudstone-sandstone interface). $\mathrm{C}$ - block diagram illustrating sandstone-mudstone interface with sedimentary features and stable burrows made by T. pedum in mudstone unit. Gaps denoted by broken lines represent the part of the burrows where priapulid worm emerged on the surface close to sediment-water interface before re-entering the unconsolidated sediments. - D deposition of successive thin layers of sandstone passively fills the burrows formed in the underlying mudstone. $\cdot \mathrm{E}$ - continuous deposition of sandstone over the in-filled burrows seals and preserves them on the sole of the sandstone. $-\mathrm{F}$ - mechanical or natural exposure unearths burrows of T. pedum preserved as positive hyporelief whereas gaps denoting emergence of priapulid worms on the surface are merged with the sediments of overlying sandstone. boundary (Jensen et al. 2000, Jensen 2003, Buatois 2018); whereas in others, Treptichnus pedum is recorded in the early Cambrian and survived up to the Ordovician (Seilacher 2007). A solitary record shows the presence Treptichnus in recent times (see Muñiz Guinea et al. 2014); therefore, the group has a long temporal range (Wilson et al. 2012). Vannier et al. (2010) demonstrated that the treptichnid burrows were made by priapulids or priapulids-like worms. A sharp decrease of T. pedum is attributed to the extinction/replacement of such priapulids (Conway-Morris 1977). In the geological history, the Precambrian-Cambrian boundary is the only stratigraphical boundary which is based on the FAD of a trace fossil (T. pedum; Brasier et al. 1994, Landing 1994, Peng et al. 2012). However, it has been advocated that T. pedum alone should not be considered as the marker trace fossil for Precambrian-Cambrian boundary/transition; rather, 'Treptichnus pedum Ichnofossil Assemblage Zone' would be more appropriate for demarcating the boundary (Narbonne et al. 1987, Landing et al. 2013, Laing et al. 2016, Buatois 2018).

Precambrian-Cambrian boundary/transition within the Marwar Basin is widely debated for its existence in the argillo-arenaceous succession of the Nagaur Group or in the underlying carbonate succession of the Bilara Group. On the basis of microfossils data, it was suggested that the boundary should be within the Bilara Group (Prasad et al. 2010). This has been questioned by Hughes (2016) on the basis of quality of microfossils illustrated in the paper of Prasad et al. (2010). Stable carbon isotope data has been used to argue suggesting that the Precambrian-Cambrian boundary lies within the carbonate succession, i.e. the Bilara Group of the Marwar Supergroup (Pandit et al. 2001, Maheshwari et al. 2003, Mazumdar \& Bhattacharya 2004, Mazumdar \& Strauss 2006, Ansari et al. 2018), however, on the basis of trace fossils assemblage, especially T. pedum, Rusophycus, Cruziana, Diplichnites, Chondrites and Monomorphichnus, it is believed that the Nagaur Sandstone is early Cambrian in age (Kumar \& Pandey 2008, 2010; Srivastava 2012a; Pandey et al. 2014; Singh et al. 2014a). Data presented here suggest that the Nagaur Sandstone in the Marwar Supergroup is the most promising succession to study the Precambrian-Cambrian boundary in the peninsular India, if FAD of T. pedum and 'Treptichnus pedum Ichnofossil Assemblage Zone' is established in the hitherto underlying unexplored vast thickness of Nagaur Sandstone.

\section{Conclusions}

1) The Treptichnus pedum marks the first infaunal activity in the Nagaur Sandstone and also represent the appearance of complex burrows pattern in the early biosphere. 
2) The Nagaur Sandstone has all the signatures which positively support the hierarchy of evolutionary trend as it demonstrates the succession from simple burrow to complex burrow, followed by miscellaneous track and trails of arthropods. The hierarchy order of such biozone is correlative with the Mackenzie Mountain, Canada.

3) Statistical analysis especially the hierarchical cluster analysis suggest that all the individuals $(\mathrm{N}=291)$ belong to the same species.

4) The burrow-producing animal lived under the thin layer of sand and occasionally protruded out of the sediment. The gaps between the two consecutive segments denote the phase of life when it came out of the sediment covering.

5) On the global scale, ichnospecies $T$. pedum is strictly found in siliciclastic sediments; it also holds true for the Nagaur Group where T. pedum is confined to Nagaur Sandstone-Siltstone alteration. The present study reiterates that the First Appearance Datum (FAD) is yet to be documented in the vast thickness of the Nagaur Sandstone which underlies the exposed succession at Dulmera locality in Bikaner District of Rajasthan.

\section{Acknowledgements}

Critical comments and suggestions of Luis A. Buatois, Radek Mikuláš and an anonymous reviewer of the journal helped us improve the manuscript. Suggestions of Sören Jensen, David Bottjer and Nigel Hughes on the earlier drafts of this manuscript are gratefully acknowledged. Insightful discussions with I.B. Singh and Satyendra Singh in understanding the facies and petrology of the ichnofossil bearing sandstone helped us develop our arguments. N.R. Mckenzie helped us with linguistic improvements. This is a contribution to the IGCP 587. We are thankful to the Director, Birbal Sahni Institute of Palaeosciences for providing the facilities to complete this work and permission to publish it (RDCC/2016-17/51). SA is thankful to CSIR for financial assistance in the form of SRF Fellowship (09/528/ (0019)/2013 EMR-I).

\section{References}

Ahmad, S. \& Kumar, S. 2014. Trace fossil assemblage from the Nagaur Group, Western India. Journal of the Palaeontological Society of India 59(2), 231-246.

Almond, J.E., Buatois, L.A., Gresse, P.G. \& Germs, G.J.B. 2008. Trend in metazoan body size, burrowing behaviour and ichnodiversity across Precambrian-Cambrian boundary: ichnoassemblages from the Vanrhynsdorp Group of South Africa. Conference Programs and Abstracts, $15^{\text {th }}$ Biennial Meeting of the Palaeontological Society of Southern Africa: Matjiesfontein, 15-20.
Alpert, S.P. 1977. Trace fossils and the basal Cambrian boundary, 1-8. In Crimes, T.P. \& HARPer, J.C. (eds) Trace fossils 2. Geological Journal Special Issue 9. Seel House Press, Liverpool.

Ansari, A.H., Pandey, S.K, Sharma, M., Agrawal, S. \& KumAR, Y. 2018. Carbon and oxygen isotope stratigraphy of the Ediacaran Bilara Group, Marwar Supergroup, India: Evidence for high amplitude carbon isotopic negative excursions. Precambrian Research 308, 75-91.

DOI 10.1016/j.precamres.2018.02.002

Babcock, L.E., Peng, S., Zhu, M., Xiao, S. \& Ahlberg, P. 2014. Proposed reassessment of the Cambrian GSSP. Journal of African Earth Sciences 98, 3-10.

DOI 10.1016/j.jafrearsci.2014.06.023

BALDWIN, C.T. 1977. Stratigraphy and facies association of trace fossils in some Cambrian and Ordovician rocks of northwestern Spain, 9-40. In CRIMES, T.P. \& HARPER, J.C. (eds) Trace Fossils 2. Geological Journal Special Issue 9. Seel House Press, Liverpool.

Banks, N.L. 1970. Trace fossils from the Late Precambrian and Lower Cambrian of Finnmark, Norway, 19-34. In CRImes, T.P. \& Harper, J.C. (eds) Trace Fossils. Geological Journal Special Issue 3. Seel House Press, Liverpool.

Bottjer, D.J., Hagadorn, J.W. \& Dornbos, S.Q. 2000. The Cambrian substrate revolution. GSA Today 10, 1-8.

Brasier, M., Cowie, J. \& Taylor, M. 1994. Decision on the Precambrian-Cambrian boundary stratotype. Episodes 17, 3-8.

Bromley, R.G. 1990. Trace Fossils: Biology and Taphonomy. 280 pp. Unwin Hyman, London.

Bromley, R.G. 1996. Trace fossils: Biology, taphonomy and applications. $361 \mathrm{pp}$. Chapman and Hall, London. DOI 10.1007/978-1-4899-2875-7

Bryant, I.D. \& Pickerill, R.K. 1990. Lower Cambrian trace fossils from the Buen Formation of central North Greenland: preliminary observations, 44-62. In PeEL, J.S. (ed.) Lower Cambrian Trace Fossils from Greenland. 147 pp. Grønlands Geologiske Undersøgelse, Rapport.

Buatols, L.A. 2018. Treptichnus pedum and the EdiacaranCambrian boundary: significance and caveats. Geological Magazine 155(1), 174-180. DOI 10.1017/S0016756817000656

Buators, L.A. \& Mángano, M.G. 2011. Ichnology: OrganismSubstrate interactions in Space and Time. 358 pp. Cambridge University Press, Cambridge. DOI 10.1017/CBO9780511975622

Buatois, L.A., Almond, J. \& Germs, G.J.B. 2013. Environmental tolerance and range offset of Treptichnus pedum: Implications for the recognition of the Ediacaran-Cambrian boundary. Geology 41(4), 519-522. DOI 10.1130/G33938.1

Buatois, L.A., Almond, J., Gresse, P. \& Germs, G.J.B. 2007. The elusive Proterozoic-Cambrian boundary: ichnologic data from the Vanrhynsdorp Group of South Africa, 8-9. In Zonneveld, J.P. \& GingRAs, M.K. (eds) $9^{\text {th }}$ International Ichnofabric Workshop: Abstracts with Program, Calgary.

Buatois, L.A., Mángano, M.G. \& Maples, C.G. 1997. The paradox of non-marine ichnofaunas in tidal rhythmites: integrating sedimentologic and ichnologic data from the Late 
Carboniferous of eastern Kansas, USA. Palaios 12(5), 467-481. DOI $10.2307 / 3515384$

Chauhan, D.S., Ram, B. \& Ram, N. 2004. Jodhpur Sandstone: a gift of ancient beaches to western Rajasthan. Journal of the Geological Society of India 64, 265-276.

Conway Morris, S. 1977. Fossil priapulid worms. Special Papers in Palaeontology 20, 1-95.

Corsetti, F.A. \& Hagadorn, J.W. 2000. Precambrian-Cambrian transition: Death Valley, United States. Geology 28(4), 299-302. DOI 10.1130/0091-7613(2000)28<299:PTDVUS>2.0.CO;2

Crimes, T.P. \& Anderson, M.M. 1985. Trace fossils from Late Precambrian-Early Cambrian strata of south-eastern Newfoundland (Canada): temporal and environmental implications. Journal of Paleontology 59(2), 310-343.

Crimes, T.P. \& Germs, G.J.B. 1982. Trace fossils from the Nama Group (Precambrian-Cambrian) of Southwest Africa (Namibia). Journal of Paleontology 56(4), 890-907.

Crimes, T.P., LegG, I., Marcos, A. \& Arboleya, M. 1977. ?Late Precambrian-Lower Cambrian trace fossils from Spain, 91-138. In Crimes, T.P. \& HARPER, J.C. (eds) Trace fossils 2. Geological Journal Special Issue 9. Seel House Press, Liverpool.

DAILY, B. 1972. The base of the Cambrian and the first Cambrian faunas, 13-42. In Jones, J.B. \& McGowran, B. (eds) Stratigraphic problems of the Late Precambrian and Early Cambrian, University of Adelaide, Centre for Precambrian Research Special Papers 1.

Droser, M.L., Gehling, J.G. \& Jensen, S. 1999. When the worm turned: Concordance of Early Cambrian ichnofabric and trace-fossil record in siliciclastic rocks of South Australia. Geology 27(7), 625-628.

DOI 10.1130/0091-7613(1999)027<0625:WTWTCO >2.3.CO;2

Droser, M.L., Jensen, S. \& Gehling, J.G. 2002. Trace fossils and substrates of the terminal Proterozoic-Cambrian transition: Implications for the record of early bilaterians and sediment mixing. Proceedings of National Academy of Sciences 99(20), 12572-12576. DOI 10.1073/pnas.202322499

DzIK, J. 2005. Behavioral and anatomical unity of the earliest burrowing animals and the cause of the "Cambrian explosion". Paleobiology 31(3), 503-521.

DOI 10.1666/0094-8373(2005)031[0503:BAAUOT]2.0.CO;2

Elliott, D.K. \& Martin, D.L. 1987. A new trace fossil from the Cambrian Bright Angel Shale, Grand Canyon, Arizona. Journal of Paleontology 61(4), 641-648.

DOI 10.1017/S0022336000028997

EVERITT, B.S. 1978. Graphical techniques for multivariate data. 117 pp. Heinemann Educational Books Limited, London.

Fedonkin, M.A. 1983. Nonskeletal fauna of the Podolian Pridnyestrovya, 128-139. In Velikanov, V.A., Aseeva, E.A. \& Fedonkin, M.A. (eds) The Vendian of the Ukraine. Akademiya Nauk Ukrainskoy SSSR, Naukova Dumka, Kiev.

Fillion, D. \& Pickerill, R.K. 1990. Ichnology of the Upper Cambrian? to Lower Ordovician Bell Island and Wabana groups of eastern Newfoundland, Canada. Palaeontographica Canadiana 7, 1-119.

Føyn, S. \& Glaessner, M.F. 1979. Platysolenites, other animal fossils, and the Precambrian-Cambrian transition in Norway. Norsk Geologisk Tidsskrift 59, 25-46.

Fritz, W.H. 1980. International Precambrian-Cambrian boundary working group's 1979 field study to Mackenzie Mountains, North-western Territories, Canada. Geological Survey of Canada 80(1A), 41-45.

Fritz, W.H., Narbonne, G.M. \& Gordey, S.P. 1983. Strata and trace fossils near the Precambrian-Cambrian boundary, Mackenzie, Selwyn, and Wernecke mountains, Yukon and Northwest territories. Geological Survey of Canada 83(1B), 365-375.

Gehling, J.G., Jensen, S., Droser, M.L., Myrow, P.M. \& Narbonne, G.M. 2001. Burrowing below the basal Cambrian GSSP, Fortune Head, Newfoundland. Geological Magazine 138, 213-218. DOI 10.1017/S001675680100509X

Germs, G.J.B. 1972. Trace fossils from the Nama Group, SouthWest Africa. Journal of Paleontology 46(6), 864-870.

Geyer, G. \& Uchman, A. 1995. Ichnofossil assemblages from the Nama Group (Neoproterozoic-Lower Cambrian) in Namibia and the Proterozoic-Cambrian boundary problem revisited. Beringeria Special Issue 2, 175-202.

Glaessner, M.F. 1969.Trace fossils from the Precambrian and basal Cambrian. Lethaia 2(4), 369-393.

DOI 10.1111/j.1502-3931.1969.tb01258.x

Gregory, L.C., Meert, J.G., Bingen, B.H., Pandit, M.K. \& Torsvik, T.H. 2009. Paleomagnetic and geochronologic study of Malani Igneous Suite, NW India: implications for the configuration of Rodinia and the assembly of Gondwana. Precambrian Research 170, 13-26.

DOI 10.1016/j.precamres.2008.11.004

Grotzinger, J., Bowring, S.A., SAYlor, B.Z. \& Kaufman, A.J. 1995. Biostratigraphic and geochronologic constraints on early animal evolution. Science 270, 598-604.

DOI 10.1126/science. 270.5236 .598

Hofmann, R., Mángano, M.G., Elicki, O. \& Shinaq, R. 2012. Paleoecologic and biostratigraphic significance of trace fossils from shallow-marginal-marine environment from the middle Cambrian (Stage 5) of Jordan. Journal of Paleontology 86, 831-955. DOI 10.1666/11-129R1.1

Högström, A.E., Jensen, S., Palacios, T. \& Ebbestad, J.O.R. 2013. New information on the Ediacaran-Cambrian transition in the Vestertana Group, Finnmark, northern Norway, from trace fossils and organic-walled microfossils. Norwegian Journal of Geology 93, 95-106.

Hughes, N.C. 2016. The Cambrian palaeontological record of the Indian subcontinent. Earth-Science Reviews 159, 428-461. DOI 10.1016/j.earscirev.2016.06.004

Hughes, N.C., Sell, B.K., English, L.T., Myrow, P.M. \& Singh, B.P. 2013. Cambrian trace fossils from the Parahio Formation (Tethyan Himalaya) in its type section and elsewhere. Journal of the Palaeontological Society of India 58(2), 175-193.

Jensen, S. 1997. Trace fossils from the Lower Cambrian Mickwitzia sandstone, south-central Sweden. Fossils and Strata 42, 1-110.

Jensen, S. 2003. The Proterozoic and Earliest Cambrian Trace Fossil Record; Patterns, Problems and Perspectives. Integrative and Comparative Biology 43, 219-228.

DOI 10.1093/icb/43.1.219 
Jensen, S. \& Grant, S.W.F. 1998. Trace fossils from the Dividalen Group, northern Sweden: implications for Early Cambrian biostratigraphy of Baltica. Norsk Geologisk Tidsskrift 78, 305-317.

Jensen, S. \& Mens, K. 2001. Trace fossils Didymaulichnus cf. tirasensis and Monomorphichnus isp. from the Estonian Lower Cambrian, with a discussion on the Early Cambrian ichnocoenoses of Baltica. Proceedings of the Estonian Academy of Sciences, Geology 50, 75-85.

Jensen, S. \& Runnegar, B.N. 2005. A complex trace fossil from the Spitskop Member (terminal Ediacaran-? Lower Cambrian) of southern Namibia. Geological Magazine 142, 561-569. DOI $10.1017 / \mathrm{S} 0016756805000853$

Jensen, S., Droser, M.L. \& Gehling, J.G. 2006. A critical look at the Edicaran trace fossil record, 115-157. In Kaufman, J., XIAO, S. (eds), Neoproterozoic Geobiology and Paleobiology, Topics in Geobiology 27. Springer, Dordrecht.

Jensen, S., Gehling, J.G. \& Droser, M.L. 1998. Ediacara-type fossils in Cambrian sediments. Nature 393, 567-569. DOI 10.1038/31215

Jensen, S., Högström, A.E., Høyberget, M., Meinhold, G., Palacios, T., Taylor, W.L., Ebbestad, J.O.R. \& Agić, H. 2017. Trace fossils across the Ediacaran Cambrian boundary on the Digermulen Peninsula, Arctic Norway. International Symposium on the Ediacaran Cambrian Transition, St John's, Newfoundland, Canada. Abstract Volume, 48.

Jensen, S., Saylor. B.Z., Gehling. J.G. \& Germs, G.J.B. 2000. Complex trace fossils from Terminal Proterozoic of Namibia. Geology 28(2), 144-146. DOI 10.1130/0091-7613(2000)28<143:CTFFTT $>2.0 . C O ; 2$

Kumar, S. \& Pandey, S.K. 2008. Discovery of trilobite trace fossils from the Nagaur Sandstone, the Marwar Supergroup, Bikaner District, Rajasthan. Current Science 94(8), 1081-1084.

Kumar, S. \& PAndey, S.K. 2010. Trace fossils from the Nagaur Sandstone, Marwar Supergroup, Dulmera area, Bikaner district, Rajasthan, India. Journal of Asian Earth Sciences 38, 77-85. DOI 10.1016/j.jseaes.2009.10.003

Laing, B., Buatois, L.A., Mángano, M. \& Narbonne, G. 2016. Redefining the Treptichnus pedum Ichnofossil Assemblage Zone: a critical reassessment of the Ediacaran-Cambrian boundary, 262. In Baucon, A., Neto de Carvalho, V., Rodrigues, J. (eds) ICHNIA 2016, Idanha-a-Nova, Portugal. Abstract Book International Ichnological Association.

Landing, E. 1994. Precambrian-Cambrian Boundary Global Stratotype ratified and a new perspective of Cambrian time. Geology 22, 179-182.

DOI 10.1130/0091-7613(1994)022<0179: $\mathrm{PCBGSR}>2.3 . \mathrm{CO} ; 2$

Landing, E., Geyer, G., Brasier, M.D. \& Bowring, S.A. 2013. Cambrian evolutionary radiation: context, correlation, and chronostratigraphy - overcoming deficiencies of the first appearance datum (FAD) concept. Earth-Science Reviews 123, 133-172. DOI 10.1016/j.earscirev.2013.03.008

LEgG, I.C. 1985. Trace fossil from middle Cambrian deltaic sequence, North Spain, 151-165. In Curran, H.A. (ed.) Biogenic structure: Their Use in Interpreting Depositional environment. SEPM, Special Publication 35.
LI, R. 1993. Trace fossils and ichnofacies of Middle Ordovician Gongwusu Formation, Zhuozishan, Inner Mongolia. Acta Palaeontologica Sinica 32(1), 88-104.

LiÑÁn, E. 1984. Los icnofósiles de la Formación Torreárboles (¿Precámbrico?-Cámbrico inferior) en los alredodores de Fuente de Cantos, Badajoz. Cuadernos do Laboratorio Xeologico de Laxe 8, 47-74.

MacNaughton, R.B. \& Narbonne, G.M. 1999. Evolution and Ecology of Neoproterozoic-Lower Cambrian Trace Fossils, NW Canada. Palaios 14, 97-115. DOI 10.2307/3515367

Maheshwari, A., Sial, A.N. \& Mathur, S.C. 2003. Carbon and Oxygen isotope profiles from the Terminal Pre-Cambrian Marwar Supergroup, Rajasthan, India. Carbonate Evaporite 18(1), 268-276. DOI 10.1007/BF03178383

Mángano, M.G. \& Buatois, L.A. 1999. Feeding adaptations, 458-465. In Singer, R. (ed.) Encyclopedia of Paleontology 1. Fitzroy Dearborn Publishers, Chicago.

Mángano, M.G. \& Buatois, L.A. 2003. Rusophycus leifeirikssoni en la Formación Campanario: Implicancias paleobiológicas, paleoecológicas y paleoambientales, 65-84. In BuAToIs, L.A. \& Mángano, M.G. (eds) Icnología: Hacia una convergencia entre geología y biología. Publicación Especial de la Asociación Paleontológica Argentina, 9.

Mángano, M.G. \& Buatois, L.A. 2017. The Cambrian Revolutions: Trace-fossil record, timing, links and geobiological impact. Earth-Science Reviews 173, 96-108.

DOI 10.1016/j.earscirev.2017.08.009

Mángano, M.G., Buatois, L.A. \& Macnaughton, R.B. 2012. Ichnostratigraphy. Developments in Sedimentology 64, 195-212. DOI 10.1016/B978-0-444-53813-0.00007-1

Mazumdar, A. \& Bhattacharya, S.K. 2004. Stable isotopic study of late Neoproterozoic-Early Cambrian (?) sediments from Nagaur-Ganganagar Basin, western India; possible signatures of global and regional C-isotopic events. Geochemical Journal 38, 163-175. DOI 10.2343/geochemj.38.163

Mazumdar, A. \& Strauss, H. 2006. Sulfur and Strontium isotopic compositions of carbonate and evaporate rocks from the late Neoproterozoic-early Cambrian Bilara Group: Constraints on intra-basinal correlation and global sulphur cycle. Precambrian Research 149, 217-230.

DOI 10.1016/j.precamres.2006.06.008

McKenzie, N.R., Hughes, N.C., Myrow, P.M., Xiao, S. \& Sharma, M. 2011. Correlation of Precambrian-Cambrian sedimentary successions across northern India and the utility of isotopic signatures of Himalayan lithotectonic zones. Earth and Planetary Science Letters 312, 471-483. DOI 10.1016/j.eps1.2011.10.027

Miller, S.A. 1889. North American Geology and Palaeontology for the Use of Amateurs, Students, and Scientists. 718 pp. Press of Western Methodist Book Concern, Cincinnati.

Muñiz Guinea, F., Mángano, M.G., Buatois, L.A., Podeniene, V., Gámez Vintaned, J.A. \& Mayoral Alfaro, E. 2014. Compound biogenic structures resulting from ontogenetic variation: An example from a modern dipteran. [Estructuras biogénicas compuestas resultantes de variación ontogenética: un ejemplo para un díptero moderno]. Spanish Journal of Palaeontology 29(1), 83-94. 
Narbonne, G.M., Myrow, P., Landing, E. \& Anderson, M.M. 1987. A candidate Stratotype for the Precambrian-Cambrian boundary, Fortune Head, Burin Peninsula, southeastern Newfoundland. Canadian Journal Earth Science 24, 1277-1293. DOI 10.1139/e87-124

Nowlan, G.S., Narbonne, G.M. \& Fritz, W.H. 1985. Small Shelly fossils and trace fossils near the Precambrian-Cambrian boundary in the Yukon Territory, Canada. Lethaia 18, 233-256. DOI 10.1111/j.1502-3931.1985.tb00701.x

OrŁowski, S. 1989. Trace fossils in the Lower Cambrian sequence in the Świętokrzyskie Mountains, Central Poland. Acta Palaeontologica Polonica 34, 211-231.

OrŁowsKi, S. \& ŻYLIŃSKA, A. 1996. Non-arthropod burrows from the Middle and Late Cambrian of the Holy Cross Mountains, Poland. Acta Palaeontologica Polonica 41(4), 385-409.

Paczesna, J. 1985. Skamienialosci sladowne górnego wendu i dolnego kambru poludniowej Lubelszczyzny. Kwartalnik Geologiczny 29, 255-270.

Paczesna, J. 1986. Upper Vendian and Lower Cambrian ichnocoenoses of the Lubin region. Instytut Geologiczny Biuletyn 355, 31-47.

PaLis, V.M. 1976. Remains of soft-bodied animals and trace fossils from the Upper Precambrian and Lower Cambrian of Podolia, 63-76. In RyabenKo, V.A. (ed.) Paleontologiya i stratigrafiya verkhnego dokembriya i nizhnego paleozoya jugo-zapadna vostochno-evropejskoj platformy [Paleontology and Stratigraphy of Upper Precambrian and Lower Paleozoic of the South-West of Eastern-European Platform]. Naukova Dumka, Kiev. [in Russian]

Paliu, V.M., Posti, E. \& Fedonkin, M.A. 1983. Soft-bodied Metazoa and animal trace fossils in the Vendian and early Cambrian, 56 - 94. In Urbanek, A. \& RozAnov, A.Y. (eds) Upper Precambrian and Cambrian Palaeontology of the East-European Platform. Publ. House Wydawnictwa, Warszawa.

Pandey, D.K., Uchman, A., Kumar, V. \& SheKhawat, R.S. 2014. Cambrian trace fossils of the Cruziana Ichnofacies from the Bikaner-Nagaur Basin, north western Indian Craton. Journal of Asian Earth Sciences 81, 129-141.

DOI 10.1016/j.jseaes.2013.11.017

Pandit, M.K., Sial, A.N., Jamrani, S.S. \& Ferreira, V.P. 2001. Carbon isotopic profiles across the Bilara Group rocks of Trans-Aravalli Marwar Supergroup in western India: implications for Neoproterozoic-Cambrian transition. Gondwana Research 4, 387-394.

DOI 10.1016/S1342-937X(05)70338-5

PARChA, S.K. \& Singh, B.P. 2005. Palaeoecological significance of ichnofossils from the Early Cambrian succession of the Spiti Valley, Tethys Himalaya, India. Current Science 88(1), $158-162$.

Parcha, S.K. \& Singh, B.P. 2010. Stratigraphic significance of the Cambrian ichnofauna of the Zanskar region of Ladakh Himalaya, India. Journal of the Geological Society of India 75, 503-517. DOI 10.1007/s12594-010-0040-x

Pareek, H.S. 1984. Pre-Quaternary Geology and Mineral resources of northwestern Rajasthan. Memoir Geological Survey of India 115, 1-95.
Peng, S., Babcock, L.E. \& Cooper, R.A. 2012. The Cambrian Period, 437-488. In Gradstein, F.M., OGG, J.G., Schmitz, M.D. \& OGG, G.M. (eds) The Geologic Time Scale, Volume 1. Elsevier, Amsterdam.

Prasad, B., Asher, R. \& Borgohai, B. 2010. Late Neoproterozoic (Ediacaran)-Early Palaeozoic (Cambrian) acritarchs from the Marwar Supergroup, Bikaner-Nagaur basin, India. Journal of the Geological Society of India 75, 415-431. DOI 10.1007/s12594-010-0038-4

Regalia, G.M. \& Herrera, H.E. 1981. Phycodes aff. pedum (traza fósil) en estratos cuaricitos de San Manuel, Sierras septentrionales de la Provincia de Buenos Aires. Revista de la Asociacion Geologica Argentina 36(3), 257-261.

Richter, R. 1850. Aus der thüringischen Grauwacke. Deutsche Geologische Gesellschaft, Zeitschrift 2, 198-206.

Seilacher, A. 1955. Spuren und Fazies im Unterkambrium, 373-399. In Schindewolf, O.H. \& SeIlacher, A. (eds) Beiträge zur Kenntnis des Kambriums in der Salt Range (Pakistan). Akademie der Wissenschaften und der Literatur zu Mainz, Mathematisch-Naturwissenschaftliche Klasse, Abhandlungen 10.

SeIlacher, A. 1956. Der Beginn des Kambriums als biologische Wende. Neues Jahrbuch für Geologie und Palaeontologie 103(1-2), 155-180.

Seilacher, A. 1967. Fossil behaviour. Scientific American 217, 72-80. DOI 10.1038/scientificamerican0867-72

Seilacher, A. 1969. Sedimentary rhythms and trace fossils in Paleozoic sandstones of Libya, 117-123. In KANES, W.H. (ed.) Geology, archaeology and prehistory of the southwestern Fezzan, Libya. Petroleum Exploration Society of Libya, $11^{\text {th }}$ Annual Field Conference, 1969, Tripoli.

SeIlacher, A. 1999. Oecoptychius Rätsel. Fossilien 3, 131.

Seilacher, A. 2007. Trace fossil analysis. 226 pp. Springer, Berlin.

Seilacher, A. \& Hemleben, E. 1966. Beitrage zur Sedimentation und Fos-silfiihrung des Hunsriickschiefers 14. Spurenfauna und Bildungstiefe der Hunsriickschiefer (Unterdevon). Notizblatt des Hessichen Lande-samtes fur Bodenforschung zu Wiesbaden 94, 40-53.

Seilacher, A. \& Pflüger, F. 1994. From biomats to benthic agriculture: a biohistoric revolution, 97-105. In KRUMBEIN, W., Paterson, D.M. \& Stal, L.J. (eds) Biostabilization of sediments. Bibliotheks und Informationssystem der Universität Oldenburg, Oldenburg.

Seilacher, A., Buatois, L.A. \& Mángano, M.G. 2005. Trace fossils in the Ediacaran-Cambrian transition: Behavioral diversification, ecological turnover and environmental shift. Palaeogeography Palaeoclimatolgy Palaeoecology 227, 323-356. DOI 10.1016/j.palaeo.2005.06.003

Shah, S.K. \& Sudan, C.S. 1983. Trace fossils from the Cambrian of Kashmir and their stratigraphic significance. Journal of the Geological Society of India 24(4), 194-202.

Sharma, M. \& PANDEY S.K. 2011. Ichno-fossils and microfossils from the Precambrian-Cambrian Marwar Supergroup, India. World Conference on Paleontology and Stratigraphy 2011, 73.

Sharma, M., Pandey, S.K., Ahmad, S., Kumar, K. \& AnSARI, A.H. 2018. Observations on the ichnospecies Mono- 
morphichnus multilineatus from the Nagaur Sandstone (Cambrian Series 2-Stage 4), Marwar Supergroup, India. Journal of Earth System Sciences.

DOI 10.1007/s12040-018-0973-9

Singh, B.P., Bhargava, O.N., Chuabey, R.S. \& Kishore, N. 2014a. Ichnology and depositional environment of the Cambrian Nagaur Sandstone (Nagaur Group) along the Dulmera section, Bikaner Nagaur Basin, Rajasthan. Acta Geologica. Sinica 88, 1665-1680. DOI 10.1111/1755-6724.12336

Singh, B.P., Bhargava, O.N., Naval, K. \& Ahluwalia, A.D. 2013. Arthropod from the Bikaner-Nagaur Basin, Peninsular India. Current Science 104(6), 706-707.

Singh, B.P., Bhargava, O.N., Sharma, C.A., Chaubey, R.S., Prasad, S.K., Negi, R.S. \& Kishore, N. 2017. Treptichnus Ichnogenus from the Cambrian of India and Bhutan: Its relevance to the Precambrian-Cambrian boundary. Journal of the Palaeontological Society of India 62(1), 39-50.

Singh, B.P., Lokho, K., Kishore, N. \& Virmani, N. 2014b. Early Cambrian Ichnofossils from the Mussoorie Syncline and revision of trace fossil biozonation of the Lesser Himalaya, India. Acta Geologica Sinica (English Edition) 88(2), 380-393. DOI 10.1111/1755-6724.12203

Singh, I.B. \& RAI, V. 1983. Fauna and biogenic structures in the Krol-Tal succession (Vendian-Early Cambrian), Lesser Himalaya: their biostratigraphic and palaeoecological significance. Journal of the Palaeontological Society of India 28, 67-90.

Sour-Tovar, F., Hagadorn, J.W. \& Huitron-Rubio, T. 2007. Ediacaran and Cambrian index fossils from Sonora, Mexico. Palaeontology 50(1), 169-175. DOI 10.1111/j.1475-4983.2006.00619.x

Srivastava, P. 2012a. Treptichnus pedum: An Ichnofossil representing Ediacaran-Cambrian Boundary in the Nagaur
Group, the Marwar Supergroup, Rajasthan, India. Proceedings of Indian National Science Academy 78(2), 161-169.

Srivastava, P. 2012b. Problematic worms and Priapulid-like Fossils from the Nagaur Group, the Marwar Supergroup, Western Rajasthan, India. Ichnos 19(3), 156-164. DOI 10.1080/10420940.2012.702606

Tarhan, L.G. \& Droser, M.L. 2014. Widespread delayed mixing in early to middle Cambrian marine shelfal settings. Palaeogeography, Palaeoclimatology, Palaeoecology 399, 310-322. DOI 10.1016/j.palaeo.2014.01.024

Vannier, J., Calandra, I., Gaillard, C. \& ŻylińsKa, A. 2010. Priapulid worms: Pioneer horizontal burrowers at the Precambrian-Cambrian boundary. Geology 38, 711-714. DOI 10.1130/G30829.1

Walter, M.R., Elphistone, R. \& Heys, G.R. 1989. Proterozoic and Early Cambrian trace fossils from the Amadeus and Georgina Basins, central Australia. Alcheringa 13, 209-256. DOI 10.1080/03115518908527821

WANG, Y. \& WANG, P. 2006. Ichnofossil Treptichnus from the Kaili Formation at Taijiang County, Guizhou Province. Geological Review 52(1), 1-10.

Wilson, J.P., Grotzinger, J.P., Fischer, W.W., Hand, K.P., JenSen, S., Knoll, A.H., Abelson, J., Metz, J.M., McLoughlin, N., Cohen, P.A. \& Tice, M.M. 2012. Deep-water incised valley deposits at the Proterozoic-Cambrian boundary in southern Namibia contain abundant Treptichnus pedum. Palaios 27(4), 252-273. DOI 10.2110/palo.2011.p11-036r

YANG, S. 1994. Trace fossils from Early-Middle Cambrian Kaili Formation of Taijiang, Guizhou. Acta Palaeontologica Sinica 33(3), 350-358.

ZHU, M. 1997. Precambrian-Cambrian trace fossils from eastern Yunnan, China: implications for Cambrian explosion. Bulletin of National Museum of Natural Science 10, 275-312. 